\title{
Lipschitz conformal immersions from degenerating Riemann surfaces with L-2-bounded second fundamental forms
}

Journal Article

Author(s):

Rivière, Tristan

Publication date:

2013-01

Permanent link:

https://doi.org/10.3929/ethz-b-000063894

Rights / license:

In Copyright - Non-Commercial Use Permitted

Originally published in:

Advances in Calculus of Variations 6(1), https://doi.org/10.1515/acv-2012-0108 


\title{
Lipschitz conformal immersions from degenerating Riemann surfaces with $L^{2}$-bounded second fundamental forms
}

\author{
Tristan Rivière \\ Communicated by Giuseppe Mingione
}

\begin{abstract}
We give an asymptotic lower bound for the Willmore energy of weak immersions with degenerating conformal class. This lower bound is used in several other works. It is for instance one of the ingredients used by the author (2010) for providing an alternative proof of the one by L. Simon of the existence of a smooth torus minimizing the Willmore energy. The main result of the present paper has been independently obtained by Kuwert and Li (2010).
\end{abstract}

Keywords. Conformal differential geometry, weak immersions, Willmore surfaces, minimal surfaces, optimal surfaces, geometric PDE.

2010 Mathematics Subject Classification. 30C70, 58E15, 58E30, 49Q10, 53A30, 35R01, 35J35, 35J48, 35J50.

\section{Introduction}

In [13] the author introduced a suitable framework for proceeding to the calculus of variations of Willmore Lagrangian of a surface. To that end he introduced the space of Lipschitz immersions with $L^{2}$-bounded second fundamental forms.

Let $g_{0}$ be a reference smooth metric on $\Sigma$. One defines the Sobolev spaces $W^{k, p}\left(\Sigma, \mathbb{R}^{m}\right)$ of measurable maps from $\Sigma$ into $\mathbb{R}^{m}$ in the following way:

$$
W^{k, p}\left(\Sigma, \mathbb{R}^{m}\right)=\left\{f \text { meas. } \Sigma \rightarrow \mathbb{R}^{m}: \sum_{l=0}^{k} \int_{\Sigma}\left|\nabla^{l} f\right|_{g_{0}}^{p} d \operatorname{vol}_{g_{0}}<+\infty\right\}
$$

Since $\Sigma$ is assumed to be compact, it is not difficult to see that this space is independent of the choice we have made of $g_{0}$.

First we need to have a weak first fundamental form, that is, we need $\vec{\Phi}^{*} g_{\mathbb{R}^{m}}$ to define an $L^{\infty}$ metric with a bounded inverse. The last requirement is satisfied if we assume that $\vec{\Phi}$ is in $W^{1, \infty}(\Sigma)$ and if $d \vec{\Phi}$ has maximal rank 2 at every point with some uniform quantitative control of "how far" $d \vec{\Phi}$ is from being degenerate: 
there exists $c_{0}>0$ such that

$$
|d \vec{\Phi} \wedge d \vec{\Phi}|_{g_{0}} \geq c_{0}>0,
$$

where $d \vec{\Phi} \wedge d \vec{\Phi}$ is a 2-form on $\Sigma$ taking values into 2-vectors from $\mathbb{R}^{m}$ and given in local coordinates by $2 \partial_{x} \vec{\Phi} \wedge \partial_{y} \vec{\Phi} d x \wedge d y$. The condition (1.1) is again independent of the choice of the metric $g_{0}$. For a Lipschitz immersion satisfying (1.1) we can define the Gauss map as being the following measurable map in $L^{\infty}(\Sigma)$ taking values in the Grassmannian of oriented $m-2$-planes in $\mathbb{R}^{m}$ :

$$
\vec{n}_{\vec{\Phi}}:=\star \frac{\partial_{x} \vec{\Phi} \wedge \partial_{y} \vec{\Phi}}{\left|\partial_{x} \vec{\Phi} \wedge \partial_{y} \vec{\Phi}\right|} .
$$

We then introduce the space $\mathcal{E}_{\Sigma}$ of Lipschitz immersions ${ }^{1}$ of $\Sigma$ with bounded second fundamental form as follows :

$$
\begin{aligned}
& \mathcal{E}_{\Sigma}:=\left\{\vec{\Phi} \in W^{1, \infty}\left(\Sigma, \mathbb{R}^{m}\right): \vec{\Phi} \text { satisfies (1.1) for some } c_{0}\right. \\
&\text { and } \left.\int_{\Sigma}|d \vec{n}|_{g}^{2} d \operatorname{vol}_{g}<+\infty\right\},
\end{aligned}
$$

where $g:=\vec{\Phi}^{*} g_{\mathbb{R}^{m}}$ is the pull back by $\vec{\Phi}$ of the flat canonical metric $g_{\mathbb{R}^{m}}$ of $\mathbb{R}^{m}$ and $d \mathrm{vol}_{g}$ is the volume form associated to $g$.

It is proved in [11] that any Lipschitz immersion $\vec{\Phi}$ in $\mathcal{E}_{\Sigma}$ defines a smooth conformal structure on $\Sigma$. A conformal structure $c$ being given on the two manifold $\Sigma$ we define $\mathcal{E}_{\Sigma}^{c}$ to be the subspace of $\mathcal{E}$ made of weak immersions which are conformal with respect to $c$. The conformal class to which $c$ belongs to will be denoted by $[c]$.

Let $\vec{\Phi}$ be in $\mathcal{E}_{\Sigma}$. Denote by $\pi_{\vec{n}}$ the orthonormal projections of vectors in $\mathbb{R}^{m}$ onto the $(m-2)$-plane given by $\vec{n} \vec{\Phi}$. With these notations the second fundamental form is defined as follows: ${ }^{2}$

$$
\forall X, Y \in T_{p} \Sigma, \quad \overrightarrow{\mathbb{I}}_{p}(X, Y):=\pi_{\vec{n}_{\vec{\Phi}}} d^{2} \vec{\Phi}(X, Y) .
$$

The mean curvature vector of the immersion at $p$ is given by

$$
\vec{H}:=\frac{1}{2} \operatorname{tr}_{g}(\overrightarrow{\mathbb{I}})=\frac{1}{2}\left[\overrightarrow{\mathbb{I}}\left(\varepsilon_{1}, \varepsilon_{1}\right)+\overrightarrow{\mathbb{I}}\left(\varepsilon_{2}, \varepsilon_{2}\right)\right],
$$

where $\left(\varepsilon_{1}, \varepsilon_{2}\right)$ is an orthonormal basis of $T_{p} \Sigma$ for the metric $g_{\vec{\Phi}}$.

${ }^{1}$ We will also simply call these immersions weak immersions.

${ }^{2}$ In order to define $d^{2} \vec{\Phi}(X, Y)$ one has to extend locally the vector $X$ or $Y$ by a vector-field but it is not difficult to check that $\pi_{\vec{n}} d^{2} \vec{\Phi}(X, Y)$ is independent of this extension. 
In the present paper we are mainly interested with the Lagrangian given by the $L^{2}$-norm of the second fundamental form

$$
E(\vec{\Phi}):=\int_{\Sigma}|\overrightarrow{\mathbb{I}}|_{g}^{2} d \operatorname{vol}_{g}
$$

An elementary computation gives

$$
E(\vec{\Phi}):=\int_{\Sigma}|\overrightarrow{\mathbb{I}}|_{g}^{2} d \operatorname{vol}_{g}=\int_{\Sigma}\left|d \vec{n}_{\vec{\Phi}}\right|_{g}^{2} d \operatorname{vol}_{g}
$$

This energy $E$ can be hence seen as being the Dirichlet Energy of the Gauss map $\vec{n}_{\vec{\Phi}}$ with respect to the induced metric $g_{\vec{\Phi}}$. The Gauss Bonnet Theorem implies that

$$
E(\vec{\Phi}):=\int_{\Sigma}|\overrightarrow{\mathbb{I}}|_{g}^{2} d \operatorname{vol}_{g}=4 \int_{\Sigma}|\vec{H}|^{2} d \operatorname{vol}_{g}-4 \pi \chi(\Sigma),
$$

where $\chi(\Sigma)$ is the Euler characteristic of the surface $\Sigma$. The energy

$$
W(\vec{\Phi}):=\int_{\Sigma}|\vec{H}|^{2} d \operatorname{vol}_{g}
$$

is the so-called Willmore energy.

In [13] we studied the existence minimizers of $W$ in $\mathcal{E}_{\Sigma}$ with or without constraints on the conformal class $c$ realized by $\vec{\Phi}$. We provided in particular a new flexible approach for proving L. Simon's result of the existence of a minimizer for $\Sigma$ being the torus. Following [15] we denote

$$
\beta_{g}^{m}:=\inf \{W(\vec{\Phi}): \vec{\Phi} \text { is an immersion of the genus } g \text { closed surface }\}
$$

and

$$
\omega_{g}^{m}:=\min \left\{4 \pi+\sum_{i=1}^{p}\left(\beta_{g_{i}}^{m}-4 \pi\right): g=g_{1}+\cdots+g_{p}, 1 \leq g_{i}<g\right\} .
$$

In [1] it is proved that for $g \geq 2$

$$
\beta_{g}^{m}<\omega_{g}^{m}
$$

In [13] we gave the existence of a smooth minimizer of the Willmore energy in $\mathcal{E}_{\Sigma}$ for an arbitrary closed 2-manifold $\Sigma$ provided the conformal class defined by some minimizing sequence is not degenerating. This fact is then guaranteed by the following result which has been proved first for $m=3,4$ in [9] has also been independently proved in [6] for general $m$. 
Theorem 1.1. Let $\left(\Sigma, c_{k}\right)$ be a sequence of closed Riemann surface of genus $g$ but with degenerating conformal class $\left[c_{k}\right]$ diverging to the boundary of the Moduli Space of $\Sigma$. Let $\vec{\Phi}_{k}$ be a sequence of conformal immersions in $\mathcal{E}_{\Sigma}$. Then

$$
\liminf _{k \rightarrow+\infty} \int_{\Sigma}\left|\vec{H}_{\vec{\Phi}_{k}}\right|^{2} d \operatorname{vol}_{\vec{\Phi}_{k}^{*} g_{\mathbb{R}^{m}}} \geq \min \left\{8 \pi, \omega_{g}^{m}\right\}
$$

and the lower bound $\min \left\{8 \pi, \omega_{g}^{m}\right\}$ can be replaced by $8 \pi$ if $g=1$.

This result plays a crucial role in [3] for proving the compactness of Willmore surfaces below $\min \left\{8 \pi, \omega_{g}^{m}\right\}$ modulo the action of conformal diffeomorphisms (see [3, Theorem I.5]).

An important result in [7] asserts that $\min \left\{8 \pi, \omega_{g}^{m}\right\}$ converges to $8 \pi$ as $g$ goes to $+\infty$.

One of the main tool we are using for proving (1.4) is the following consequence of Simon's monotonicity formula with boundary which was probably known by the experts in the field but for which we still give a proof below.

Lemma 1.2. Let $\Sigma$ be a compact surface with boundary. Let $\vec{\Phi}$ be a weak immersion in $\mathcal{E}_{\Sigma}$. Then the following inequality holds:

$$
4 \pi \leq \int_{M}|\vec{H}|^{2} d \operatorname{vol}_{g}+2 \frac{\mathscr{H}^{1}(\partial M)}{d(\partial M, M)},
$$

where $\mathscr{H}^{1}(\partial M)$ is the 1-dimensional Hausdorff measure of the boundary of the immersion $\partial M$ and $d$ is the usual distance $e^{3}$ between two sets.

Observe that the inequality is optimal since it is an equality for $M$ being the flat 2-dimensional disc.

\section{The normalization procedure}

The aim of this section is to prove the "normalization" result Proposition 2.1. This result asserts that a sequence of conformal immersions $\vec{\Phi}_{k}$ of $\mathcal{E}_{\Sigma}$ realizing a degenerating sequence of conformal classes on $\Sigma$ being given, either (1.4) holds or, for any connected component $\sigma$ of the limit $\bar{\Sigma}$ topologically obtained from $\Sigma$ by removing collapsing geodesics for the constant Gauss curvature associated to $\left(\Sigma, \vec{\Phi}_{k}^{*} g_{\mathbb{R}^{m}}\right)$, one can find a sequence of Möbius transformations $\Xi_{k}$ of $\mathbb{R}^{m}$ such that, away from possibly finitely many blow up points on $\sigma$, the sequence

${ }^{3}$ Let $A$ and $B$ be two sets in $\mathbb{R}^{m}$ we define

$$
d(A, B):=\sup _{p \in A} \inf _{q \in B}|p-q|+\sup _{p \in A} \inf _{q \in B}|q-p| .
$$


of immersion $\Xi_{k} \circ \vec{\Phi}_{k}$ converges weakly on $\sigma$ in $W^{2,2} \cap\left(W^{1, \infty}\right)^{*}$ (with respect to some reference constant Gauss curvature metric) to a non-constant conformal immersion of the component $\sigma$.

The proof of this normalization result will be a consequence of Deligne-Mumford's description of the loss of compactness of the conformal class for a sequence of Riemann surfaces, the Three Point Normalization Lemma A.1, the Simon's monotonicity formula with boundary and Müller-Sverak-Hélein local control of conformal factors.

First we recall Deligne-Mumford's description of the loss of compactness of the conformal class for a sequence of Riemann surfaces with a fixed topology (see for instance [16] and [5, Proposition 5.1]). We restrict below in the presentation of the proof to the more complex case where $g(\Sigma)>1$, the case of tori is following the same lines and we shall comment it at the end of the next section.

In what follows, let $\left(\Sigma, c_{k}\right)$ be a sequence of closed Riemann surface of fixed topology $g(\Sigma)>1$. Denote by $h_{k}$ the hyperbolic metric associated to $c_{k}$. Let $\vec{\Phi}_{k}$ a sequence of immersions in $\mathcal{E}_{\Sigma}$ with uniformly bounded energy. Then, modulo extraction of a subsequence, there exists

(i) an integer $n \in \mathbb{N}^{*}$,

(ii) a sequence $\mathscr{L}_{k}=l_{k}^{i}, i=1, \ldots, N$, of finitely many pairwise disjoint simple closed geodesics of $\left(\Sigma, h_{k}\right)$ with length converging to zero,

(iii) a closed Riemann surface $(\bar{\Sigma}, \bar{c})$,

(iv) a complete hyperbolic Riemann surface $(\tilde{\Sigma}, \tilde{h})$ with $2 n$ cusps $\left\{q^{i}\right\}_{i=1, \ldots, n}=$ $\left\{\left(q_{1}^{i}, q_{2}^{i}\right)\right\}_{i=1, \ldots, n}$ - or punctures - and no boundary such that $\tilde{\Sigma}$ has been obtained topologically after removing the geodesics $\mathscr{L}_{k}$ and after closing each component of the boundary of the open surface $\Sigma \backslash \mathscr{L}_{k}$ by adding a puncture $q_{l}^{i}$ at each of these components. Moreover $\bar{\Sigma}$ is topologically equal to $\tilde{\Sigma}$ and the complex structure defined by $\tilde{h}$ on $\tilde{\Sigma} \backslash\left\{q_{i}\right\}$ extends uniquely to $\bar{c}$ (i.e. there exists a conformal diffeomorphism from the surface $\left(\tilde{\Sigma} \backslash \mathscr{L}_{k}, \tilde{h}\right)$ into $\left.\left(\bar{\Sigma} \backslash\left\{q^{i}\right\}_{i=1, \ldots, n}, \bar{c}\right)\right)$.

The surface $(\tilde{\Sigma}, \tilde{h})$ is called the nodal surface of the converging sequence and $(\bar{\Sigma}, \bar{c})$ is its renormalization. These objects are related with another by the mean of a sequence of continuous maps $\phi_{k}$ from $\left(\tilde{\Sigma} \backslash \bigcup_{i} q^{i}, \tilde{h}\right)$ into $\left(\Sigma \backslash \mathscr{L}_{k}, h_{k}\right)$ such that

(1) $\phi_{k}$ realizes a $C_{\text {loc }}^{\infty}$ diffeomorphism from $\left(\tilde{\Sigma} \backslash \bigcup_{i} q^{i}, \tilde{h}\right)$ into $\left(\Sigma \backslash \mathscr{L}_{k}, h_{k}\right)$,

(2) $\tilde{h}_{k}:=\phi_{k}^{*} h_{k}$ converges in $C_{\text {loc }}^{\infty}$ topology on $\tilde{\Sigma} \backslash \cup_{i} q^{i}$ to $\tilde{h}$.

Now, under the notations above, the aim of this section is to prove the following proposition. 
Proposition 2.1. Let $\left(\Sigma, c_{k}\right)$ be a sequence of closed Riemann surface of fixed topology $g(\Sigma)>1$ with degenerating conformal class $c_{k}$. Let $\vec{\Phi}_{k}$ be a sequence of weak immersions of $\Sigma$ with $L^{2}$-bounded second fundamental form (i.e. $\vec{\Phi}_{k} \in \mathcal{E}_{\Sigma}$ ). Then either

$$
\liminf _{k \rightarrow+\infty} \int_{\Sigma}\left|\vec{H}_{\vec{\Phi}_{k}}\right|^{2} d \operatorname{vol}_{\vec{\Phi}_{k}^{*} g_{\mathbb{R}^{m}}} \geq 8 \pi
$$

or, if this is not true, then for any connected component $\sigma$ of $\tilde{\Sigma}$ and there exists a subsequence still denoted by $\vec{\Phi}_{k}$ and a subsequence of Möbius transformations $\Xi_{k}$ of $\mathbb{R}^{m}$ such that

$$
\Xi_{k} \circ \vec{\Phi}_{k}\left(\Sigma_{k}\right) \subset B_{R}(0)
$$

and

$$
\mathscr{H}^{2}\left(\Xi_{k} \circ \vec{\Phi}_{k}\left(\Sigma_{k}\right)\right) \leq C R^{2} \sup _{k} W\left(\vec{\Phi}_{k}\right),
$$

where $R$ and $C$ only depend on $m$, and there exists at most finitely many points $\left\{a_{1}, \ldots, a_{N}\right\}$ of $\sigma$ such that, if we denote $\vec{\xi}_{k}:=\Xi_{k} \circ \vec{\Phi}_{k} \circ \phi_{k}$, then

$$
\vec{\xi}_{k} \rightarrow \vec{\xi}_{\infty} \text { weakly in } W_{\text {loc }}^{2,2}\left(\sigma \cap \tilde{\Sigma} \backslash\left\{a_{1}, \ldots, a_{N}\right\} \cup\left\{q^{1}, \ldots, q^{n}\right\}, \tilde{h}\right) \text {. }
$$

Moreover, for any compact $K \subset \sigma \cap \tilde{\Sigma} \backslash\left\{a_{1}, \ldots, a_{N}\right\} \cup\left\{q^{1}, \ldots, q_{n}\right\}$ there exists $C_{K}>0$ such that

$$
\sup _{k \in \mathbb{N}}\left\|\log \left|d \vec{\xi}_{k}\right|_{\phi_{k}^{*} h_{k}}\right\|_{L^{\infty}(K)} \leq C_{K}<+\infty,
$$

and the limit $\vec{\xi}_{\infty}$ is an element of $\mathcal{E}_{\sigma}^{\bar{c}}$ : it realizes a non-constant weak conformal immersion of $(\sigma \cap \bar{\Sigma}, \bar{c})$ into $B_{R}(0)$.

Proof. We work of course under the assumption that

$$
\int_{\Sigma}\left|\vec{H}_{\vec{\Phi}_{k}}\right|^{2} d \operatorname{vol}_{\vec{\Phi}_{k}^{*} g_{\mathbb{R}} m}<8 \pi \text {. }
$$

Let $P_{1}, P_{2}$ and $P_{3}$ be three distinct points in a given component $\tilde{\sigma}$ of the set $\tilde{\Sigma} \backslash\left\{q^{1}, \ldots, q^{n}\right\}$. We apply the Three Points Normalization Lemma A.1 to $\vec{\Phi}_{k}$ on $\Sigma$ for the three points $\phi_{k}\left(P_{1}\right), \phi_{k}\left(P_{2}\right)$ and $\phi_{k}\left(P_{3}\right)$ and we obtain the existence of a Möbius transformation $\Xi_{k}$ such that

$$
\Xi_{k} \circ \vec{\Phi}_{k}\left(\Sigma_{k}\right) \subset B_{R}(0),
$$

moreover

$$
\forall i \neq j, \quad\left|\Xi_{k} \circ \vec{\Phi}_{k} \circ \phi_{k}\left(P_{i}\right)-\Xi_{k} \circ \vec{\Phi}_{k} \circ \phi_{k}\left(P_{j}\right)\right| \geq r>0
$$

and

$$
\mathscr{H}^{2}\left(\Xi_{k} \circ \vec{\Phi}_{k}\left(\Sigma_{k}\right)\right) \leq C R^{2} \sup _{k} W\left(\vec{\Phi}_{k}\right),
$$

where $R, r$ and $C$ are positive constants depending only on $m$ since (2.6) holds. 
We denote by $\vec{\xi}_{k}$ the conformal immersion from $\left(\tilde{\Sigma}, \tilde{h}_{k}=\phi_{k}^{*} h_{k}\right)$ given by

$$
\vec{\xi}_{k}:=\Xi_{k} \circ \vec{\Phi}_{k} \circ \phi_{k}
$$

Let $\delta>0$ be a small positive constant and denote

$$
\tilde{\Sigma}_{\delta}:=\tilde{\Sigma} \backslash \bigcup_{j=1}^{n} B_{\delta}^{\bar{h}}\left(q^{j}\right)
$$

where $B_{\delta}^{\bar{h}}\left(q^{j}\right)$ is the geodesic ball in $(\bar{\Sigma}, \bar{h})$ of radius $\delta$ and center $q^{j}$. On $\tilde{\Sigma}_{\delta}$ there exists an $L^{\infty}$-bounded smooth function $l$ such that $\tilde{h}=e^{l} \bar{h}$.

To each $p \in \tilde{\Sigma}_{\delta}$ we assign $\rho_{p}>0$ such that

$$
\int_{B_{\rho_{p}}^{\tilde{h}}(p)}\left|d \vec{n}_{\vec{\xi}_{k}}\right|_{\tilde{h}_{k}}^{2} d \operatorname{vol}_{\tilde{h}_{k}}=\int_{B_{\rho_{p}}(p)}\left|d \vec{n}_{\vec{\xi}_{k}}\right|_{g_{k}}^{2} d \operatorname{vol}_{g_{k}}=8 \pi / 3,
$$

where $B_{\rho_{p}}^{\tilde{h}}(p)$ is the geodesic ball in the surface $(\tilde{\Sigma}, \tilde{h})$ of center $p$ and radius $\rho_{p}$ and where $g_{k}:=\vec{\xi}_{k}^{*} g_{\mathbb{R}^{m}}$.

We extract a Besicovitch covering: each point in $\tilde{\Sigma}_{\delta}$ is covered by at most $N$ of such balls where $N$ only depends on $\left(\tilde{\Sigma}_{\delta}, \tilde{h}\right)$. Since $\int_{\tilde{\Sigma}}|d \vec{n}|_{g_{k}}^{2} d$ vol $_{g_{k}}$ is uniformly bounded independent of $k$, the number of balls in the extracted Besicovitch covering has to be uniformly bounded independently of $k$.

Let $\left(B_{\rho_{k}^{i}}\left(p_{k}^{i}\right)\right)_{i \in I}$ be this finite covering (we shall omit now the superscript $\tilde{h}$ ). We can extract a subsequence such that $I$ is independent of $k$, such that each $p_{k}^{i}$ converges to a limit $p_{\infty}^{i}$ and each $\rho_{k}^{i}$ converges to a limit $\rho_{\infty}^{i}$. Let

$$
I_{0}:=\left\{i \in I: \rho_{\infty}^{i}=0\right\} .
$$

Let $I_{1}:=I \backslash I_{\tilde{\sim}}$. Clearly, the union of the closures of the balls, $\bigcup_{i \in I_{1}} \overline{B_{\rho_{\infty}^{i}}\left(\tilde{\sim}_{\infty}^{i}\right)}$, covers the set $\tilde{\Sigma}_{\delta}$. Because of the strict convexity of the balls (the metric $\tilde{h}$ has constant curvature either 0 or -1 ) the points in $\tilde{\Sigma}_{\delta}$ which are not contained in the union of the open balls, $\bigcup_{i \in I_{1}} B_{\rho_{\infty}^{i}}\left(p_{\infty}^{i}\right)$, cannot accumulate and therefore are isolated and hence finite. Denote

$$
\left\{a_{1}, \ldots, a_{N}\right\}:=\tilde{\Sigma}_{\delta} \backslash \bigcup_{i \in I_{1}} B_{\rho_{\infty}^{i}}\left(p_{\infty}^{i}\right) .
$$

We prove now that the following claim holds.

Claim 1. On the connected component $\tilde{\sigma}$ of $\tilde{\Sigma}$ either there exists a subsequence $k^{\prime}$ such that for any compact $K \subset \tilde{\sigma} \cap \tilde{\Sigma}_{\delta} \backslash\left\{a_{1}, \ldots, a_{N}\right\}$

$$
\sup _{k^{\prime} \in \mathbb{N}}\left\|\log \left|d \vec{\xi}_{k^{\prime}}\right|_{\tilde{h}_{k^{\prime}}}\right\|_{L^{\infty}(K)}<+\infty
$$


or for any compact $K \subset \tilde{\sigma} \cap \tilde{\Sigma}_{\delta} \backslash\left\{a_{1}, \ldots, a_{N}\right\}$

$$
\log \left|d \vec{\xi}_{k}\right|_{\tilde{h}_{k}} \rightarrow-\infty \text { uniformly on } K .
$$

Proof of Claim 1. Let $i \in I_{1}$. For such an $i$ we consider on the ball $B_{\rho_{k}^{i}}\left(p_{k}^{i}\right)$ conformal coordinates $\left(x_{k}^{i}, y_{k}^{i}\right) \in D^{2}$ for the metric $\tilde{h}_{k}$ which converge in ${ }^{\rho_{k}} C^{\infty}$-norm to conformal coordinates $\left(x^{\infty}, y^{\infty}\right)$ for $\tilde{h}$ (this is clearly possible since the sequence of metrics $\tilde{h}_{k}$ converge to $\tilde{h}$ in $C^{\infty}$-norm). Denote by $f_{k}^{i}$ the inverse of $\left(x_{k}^{i}, y_{k}^{i}\right)$ (i.e. $f_{k}^{i}$ is then a conformal diffeomorphism from $D^{2}$ into $\left.\left(B_{\rho_{k}^{i}}\left(p_{k}^{i}\right), \tilde{h}_{k}\right)\right)$. Due to conformal invariance one has

$$
\begin{aligned}
\int_{D^{2}}\left|\nabla \vec{n}_{\vec{\xi}_{k} \circ f_{k}^{i}}\right|^{2} d x d y & =\int_{B_{\rho_{k}^{\bar{h}_{j}}\left(p_{k}^{i}\right)}|d \vec{n}|_{h_{k}}^{2} d \operatorname{vol}_{h_{k}}} \\
& =\int_{\substack{B_{\rho_{k}^{i}}\left(p_{k}^{i}\right) \\
\rho^{i}}}|d \vec{n}|_{g_{k}}^{2} d \operatorname{vol}_{g_{k}}=8 \pi / 3
\end{aligned}
$$

and therefore $\vec{\xi}_{k} \circ f_{k}^{i}$ satisfies all the assumptions of Lemma A.2. We then apply Lemma A.2 and since $f_{k}^{i}$ converges in $C^{\infty}$-norm to some limiting conformal coordinates from the set $D^{2}$ into the ball $B_{\rho_{\infty}^{i}}^{\bar{h}}\left(p_{\infty}^{i}\right)$, we deduce the following alternative:

Either there exists a subsequence $k^{\prime}$ such that for any compact $K \subset B_{\rho_{\infty}^{i}}^{\bar{h}}\left(p_{\infty}^{i}\right)$

$$
\underset{k^{\prime} \in \mathbb{N}}{\limsup }\left\|\log \left|d \vec{\xi}_{k^{\prime}}\right|_{\tilde{h}_{k^{\prime}}}\right\|_{L^{\infty}(K)}<+\infty
$$

or for any compact $K \subset B_{\rho_{\infty}^{i}}^{\bar{h}}\left(p_{\infty}^{i}\right)$

$$
\log \left|d \vec{\xi}_{k}\right|_{\tilde{h}_{k}} \rightarrow-\infty \quad \text { uniformly on } K .
$$

This alternative holds on any ball

$$
B_{\rho_{\infty}^{i}}^{\bar{h}}\left(p_{\infty}^{i}\right) \subset \tilde{\Sigma},
$$

it is then clear that, since none of this ball is "separated" from the others in a given connected component of $\tilde{\Sigma}$, the claim is proved.

We are now going to prove the following claim:

Claim 2. If the second alternative of Claim 1, (2.12), holds on $\tilde{\sigma}$, then (2.1) holds:

$$
\liminf _{k \rightarrow+\infty} \int_{\Sigma}\left|\vec{H}_{\vec{\Phi}_{k}}\right|^{2} d \operatorname{vol}_{\vec{\Phi}_{k}^{*} g_{\mathbb{R}^{m}}} \geq 8 \pi
$$

Proof of Claim 2. Assuming the second alternative (2.12) of Claim 1 implies that, modulo extraction of a subsequence, the complement of $\left\{a_{1}, \ldots, a_{N}\right\}$ in $\sigma \cap \tilde{\Sigma}_{\delta}$ is collapsing to a point $Q \in B_{R}(0)$. 
Because of (2.8), among the three points $P_{1}, P_{2}$ and $P_{3}$ we need at least two $P_{j}$ to coincide with two distinct blowing points $a^{i}$, say $P_{1}=a^{1}$ and $P_{2}=a^{2}$, and such that

$$
\text { for } i=1,2, \quad \liminf _{k \rightarrow+\infty}\left|\vec{\xi}_{k}\left(P_{i}\right)-Q\right| \geq \frac{r}{2}>0
$$

Let $s>0$ such that the different balls $B_{s}^{\tilde{h}_{k}}\left(a_{i}\right)$ included in $\tilde{\Sigma}_{\delta}$ are disjoint. Consider

$$
M_{k}^{i}:=\vec{\xi}_{k}\left(B_{s}^{\tilde{h}_{k}}\left(a^{i}\right)\right)
$$

and take conformal coordinates $\left(x_{k}^{i}, y_{k}^{i}\right) \in D^{2}$ for the metric $\tilde{h}_{k}$ which converge in $C^{\infty}$-norm to conformal coordinates $\left(x^{\infty}, y^{\infty}\right)$ for $\tilde{h}$ (this is clearly possible since the sequence of metrics $\tilde{h}_{k}$ converge to $\tilde{h}$ in $C^{\infty}$-norm). Denote by $f_{k}^{i}$ the inverse of $\left(x_{k}^{i}, y_{k}^{i}\right)$ (i.e. $f_{k}^{i}$ is then a conformal diffeomorphism from $D^{2}$ into the surface $\left.\left(B_{s} \tilde{h}_{k}\left(a^{i}\right), \tilde{h}_{k}\right)\right)$. We have in these coordinates that

$$
\mathscr{H}^{1}\left(\partial M_{k}^{i}\right)=\int_{\partial D^{2}} e^{\lambda_{k}^{i}} d \theta,
$$

where $\lambda_{k}^{i}=\log \left|\partial_{\theta} \vec{\xi}_{k} \circ f_{k}^{i}\right|$. Combining the fact that $s$ is fixed and the fact that $\tilde{h}_{k}$ converges in any $C^{l}$-norm to a limiting metric $\tilde{h}$ we have that $\partial M_{k}^{i}$ stays contained in a compact subset $K$ of $\tilde{\Sigma}_{\delta} \backslash\left\{a^{1}, \ldots, a^{N}\right\}$. Since moreover $f_{k}^{i}$ and its inverse converge strongly in any $C^{l}$-norm to a limiting conformal parametrization of $B_{s}^{\tilde{h}}\left(a^{i}\right)$ and since we are assuming that the second alternative (2.12) of Claim 1 holds, we deduce that

$$
\lambda_{k}^{i} \rightarrow-\infty \text { uniformly on } \partial D^{2}
$$

Thus we have that

$$
\lim _{k \rightarrow+\infty} \mathscr{H}^{1}\left(\partial M_{k}^{i}\right)=0
$$

Consider the monotonicity formula (A.13) for $M_{k}^{1}\left(\operatorname{resp} . M_{k}^{2}\right)$ and $\vec{x}^{0}=\vec{\xi}_{k}\left(P_{1}\right)$ (resp. $\vec{x}^{0}=\vec{\xi}_{k}\left(P_{2}\right)$. For a fixed $k$ we make $t \rightarrow 0$. Since

$$
\begin{aligned}
& \frac{1}{t^{2}} \int_{M_{k}^{i} \cap B_{t}\left(\vec{x}^{0}\right)}\left\langle\vec{x}-\vec{x}^{0}, \vec{H}_{\vec{\xi}_{k}}\right\rangle d \operatorname{vol}_{g_{k}} \\
& \quad \leq\left(t^{-2} \operatorname{Area}\left(M_{k}^{i} \cap B_{t}\left(\vec{x}^{0}\right)\right)^{1 / 2} W\left(\vec{\xi}_{k}(\tilde{\Sigma}) \cap B_{t}\left(\vec{x}^{0}\right)\right)\right.
\end{aligned}
$$

and since $t^{-2} \operatorname{Area}\left(M_{k}^{i} \cap B_{t}\left(\vec{x}^{0}\right)\right)$ is uniformly bounded (from the classical monotonicity formula without boundary) and since clearly $W\left(\vec{\xi}_{k}(\tilde{\Sigma}) \cap B_{t}\left(\vec{x}^{0}\right)\right) \rightarrow 0$, we deduce that for a fixed $k$

$$
\lim _{t \rightarrow+\infty} \frac{1}{t^{2}} \int_{M_{k}^{i} \cap B_{t}\left(\vec{x}^{0}\right)}\left\langle\vec{x}-\vec{x}^{0}, \vec{H}_{\vec{\xi}_{k}}\right\rangle d \operatorname{vol}_{g_{k}}=0 .
$$


Since $\vec{x}^{0}=\vec{\xi}_{k}\left(P_{1}\right)$ belongs to the interior of $M_{k}^{i}$, using again the classical monotonicity formula without boundary for $\vec{\Phi}_{k}(\Sigma)$ we have that

$$
\liminf _{t \rightarrow 0} t^{-2} \operatorname{Area}\left(M_{k}^{i} \cap B_{t}\left(\vec{x}^{0}\right)\right) \geq \pi .
$$

It is clear that

$$
\begin{aligned}
\lim _{t \rightarrow 0}- & \frac{1}{2} \int_{\partial M_{k}^{i} \cap B_{T}\left(\vec{x}^{0}\right)} \frac{1}{\rho_{t}^{2}}\left\langle\vec{x}-\vec{x}^{0}, \vec{v}\right\rangle d l_{\partial M_{k}^{i}} \\
& =-\frac{1}{2} \int_{\partial \boldsymbol{M}_{k}^{i} \cap B_{T}\left(\vec{x}^{0}\right)} \frac{1}{\left|\vec{x}-\vec{x}^{0}\right|^{2}}\left\langle\vec{x}-\vec{x}^{0}, \vec{v}\right\rangle d l_{\partial M_{k}^{i} .}
\end{aligned}
$$

Hence by combining (2.17)-(2.19) and making $T \rightarrow+\infty$ in (A.13) gives

$$
\frac{1}{4} \int_{M_{k}^{i}}|\vec{H}|^{2} d \operatorname{vol}_{g} \geq \pi-\frac{1}{2} \int_{\partial M_{k}^{i}} \frac{1}{\left|\vec{x}-\vec{x}^{0}\right|^{2}}\left\langle\vec{x}-\vec{x}^{0}, \vec{v}\right\rangle d l_{\partial M_{k}^{i}} .
$$

Since for $i=1,2$ one has (2.15) and since the boundary of $M_{k}^{i}$ is converging to $Q$, for $k$ large enough one has

$$
\text { for } i=1,2, \forall \vec{x} \in \partial M_{k}^{i}, \quad \frac{r}{4} \leq\left|\vec{x}-\vec{x}^{0}\right| \leq 2 R .
$$

Inserting this information in (2.20) gives

$$
\text { for } i=1,2, \quad \frac{1}{4} \int_{M_{k}^{i}}|\vec{H}|^{2} d \operatorname{vol}_{g} \geq \pi-\frac{8 R}{r^{2}} \mathscr{H}^{1}\left(\partial M_{k}^{i}\right) \text {. }
$$

Since (2.16) holds and since $M_{k}^{1}$ and $M_{k}^{2}$ are disjoint, we have proved (2.1) and Claim 2 is proved.

Assuming now that (2.1) would not hold, then there exists a subsequence $k^{\prime}$ such that for the chosen component $\sigma$ of $\tilde{\Sigma}$ one has (2.5): for any compact $K$ in $\sigma \backslash\left\{a^{1}, \ldots, a^{N}\right\} \cup\left\{q^{1}, \ldots, q^{n}\right\}$ one has

$$
\underset{k^{\prime} \in \mathbb{N}}{\limsup }\left\|\log \left|d \vec{\xi}_{k^{\prime}}\right|_{\tilde{h}_{k^{\prime}}}\right\|_{L^{\infty}(K)}<+\infty .
$$

Since $\tilde{h}_{k}$ strongly converges to $\tilde{h}$ and since $\tilde{h}$ and $\bar{h}$ are comparable norms away from the punctures $\left\{q^{1}, \ldots, q_{n}\right\}$, one has

$$
\limsup _{k^{\prime} \in \mathbb{N}}\left\|\log \left|d \vec{\xi}_{k^{\prime}}\right|_{\bar{h}}\right\|_{L^{\infty}(K)}<+\infty
$$

for any compact $K$ in $\sigma \backslash\left\{a^{1}, \ldots, a^{N}\right\} \cup\left\{q^{1}, \ldots, q^{n}\right\}$. Since the area of $\vec{\xi}_{k^{\prime}}(\tilde{\Sigma})$ is uniformly bounded (see (2.9)), the $W^{1,2}$-norm of $\xi_{k}$ is uniformly bounded with respect to the norm $g_{k}=\vec{\xi}_{k}^{*} g_{\mathbb{R}^{m}}$ as well as with respect to the norm $\tilde{h}_{k}$ since these 
two metrics are conformally equivalent and one has

$$
\int_{\tilde{\Sigma}}\left|d \vec{\xi}_{k^{\prime}}\right|_{\tilde{h}_{k^{\prime}}}^{2} d \operatorname{vol}_{\tilde{h}_{k^{\prime}}} \leq C R^{2} \sup _{k} W\left(\vec{\Phi}_{k}\right) .
$$

Since $\tilde{h}_{k}$ converges strongly to $\tilde{h}$ away from the punctures, one has that for any compact $K \subset \sigma \backslash\left\{q^{1}, \ldots, q^{n}\right\}$

$$
\left.\left|\int_{K}\right| d \vec{\xi}_{k}\right|_{\tilde{h}_{k}} ^{2}-\int_{K}\left|d \vec{\xi}_{k}\right|_{\tilde{h}}^{2} \mid \rightarrow 0 .
$$

Since $\tilde{h}$ and $\bar{h}$ are conformally equivalent, one has

$$
\left.\left|\int_{K}\right| d \vec{\xi}_{k}\right|_{\tilde{h}_{k}} ^{2}-\int_{K}\left|d \vec{\xi}_{k}\right|_{\frac{2}{h}}^{2} \mid \rightarrow 0 .
$$

Combining (2.23) and (2.24) gives then that, modulo extraction of a subsequence, $\vec{\xi}_{k}$ converges weakly in $W_{\text {loc }}^{1,2}\left(\sigma \backslash\left\{q^{1}, \ldots, q_{n}\right\}\right)$ to a limit $\vec{\xi}_{\infty}$; moreover

$$
\int_{\tilde{\Sigma}}\left|d \vec{\xi}_{\infty}\right| \frac{2}{h} d \operatorname{vol}_{\bar{h}} \leq C R^{2} \sup _{k} W\left(\vec{\Phi}_{k}\right) \text {. }
$$

This last inequality implies that $\vec{\xi}_{\infty} \in W^{1,2}(\sigma, \bar{h})$. Since (2.22) holds, using very classical arguments of functional analysis - see for instance $[11$, beginning of Section VI.7.1] - one obtains the following fact:

$$
\vec{\xi}_{k} \rightarrow \vec{\xi}_{\infty} \quad \text { weakly in } W_{\text {loc }}^{2,2}\left(\sigma \backslash\left\{a^{1}, \ldots, a^{N}\right\} \cup\left\{q^{1}, \ldots, q^{n}\right\}, \tilde{h}\right) .
$$

Moreover $\vec{\xi}_{\infty}$ is a weak immersion away from $\left\{a^{1}, \ldots, a^{N}\right\} \cup\left\{q^{1}, \ldots, q^{n}\right\}$ satisfying

$$
\int_{\sigma}\left|d \vec{n}_{\vec{\xi}_{\infty}}\right| \frac{2}{h} d \operatorname{vol}_{\bar{h}} \leq \liminf _{k^{\prime} \rightarrow+\infty} \int_{\sigma}\left|d \vec{n}_{\vec{\xi}_{k^{\prime}}}\right|_{\tilde{h}_{k^{\prime}}}^{2} d \operatorname{vol}_{\tilde{h}_{k^{\prime}}}<+\infty .
$$

Using now [13, Lemma A.5] permits to extend the limit $\vec{\xi}_{\infty}$ as a conformal, possibly branched, immersion of $\sigma$. If there is one branched point, then arguing as in [13, end of Section 3] we would obtain that

$$
\begin{aligned}
8 \pi \leq W\left(\vec{\xi}_{\infty}(\sigma)\right) & \leq \liminf _{k^{\prime} \rightarrow+\infty} W\left(\vec{\xi}_{k^{\prime}}(\sigma)\right) \\
& \leq \liminf _{k^{\prime} \rightarrow+\infty} W\left(\vec{\Xi}_{k^{\prime}} \circ \vec{\Phi}_{k^{\prime}}(\Sigma)\right)=\liminf _{k^{\prime} \rightarrow+\infty} W\left(\vec{\Phi}_{k^{\prime}}(\Sigma)\right)
\end{aligned}
$$

which contradicts our assumption that (2.16) does not hold. Hence $\vec{\xi}_{\infty}$ extends to a weak conformal immersion with $L^{2}$-bounded second fundamental form all over $\sigma$. 


\section{Proof of Theorem 1.1}

First we present the case $g>1$ which is more delicate. Assume that the assumptions Theorem 1.1 are fulfilled and that (2.16) does not hold.

Let $\left(\sigma^{j}\right)_{j=1, \ldots, M}$ be the connected components of $\tilde{\Sigma}$.

Consider the sequence $\vec{\xi}_{k}:=\Xi_{k}^{1} \circ \vec{\Phi}_{k} \circ \phi_{k}$ given by Proposition 2.1 for the component $\sigma^{1}$. Let $\left\{a_{1}, \ldots, a_{N}\right\}$ be the possible blow-up points for of $\vec{\xi}_{k}$ on $\sigma^{1}$ and let $\left(q^{j}\right)_{j \in J^{1}}$ be the punctures of $\left(\sigma^{1}, \tilde{h}\right)$ and denote for any $\delta>0$

$$
\sigma_{\delta}^{1}=\sigma^{1} \backslash \bigcup_{i=1}^{N} B_{\delta}^{\bar{h}}\left(a_{i}\right) \bigcup_{j \in J} B_{\delta}^{\bar{h}}\left(q^{j}\right) .
$$

Because of the convergences (2.4) and (2.5) we have

$$
\lim _{\delta \rightarrow 0} \liminf _{k \rightarrow+\infty} W\left(\vec{\xi}_{k}\left(\sigma_{\delta}^{1}\right)\right) \geq W\left(\vec{\xi}_{\infty}\left(\sigma^{1}\right)\right) .
$$

Observe first that

$$
\forall j \neq j^{\prime} \in J, \quad \vec{\xi}_{\infty}\left(q^{j}\right) \neq \vec{\xi}_{\infty}\left(q^{j^{\prime}}\right) .
$$

Indeed if it would not be the case, using the Li Yau inequality we would obtain

$$
\begin{aligned}
8 \pi \leq W\left(\vec{\xi}_{\infty}\left(\sigma^{1}\right)\right) & \leq \liminf _{k^{\prime} \rightarrow+\infty} W\left(\vec{\xi}_{k^{\prime}}\left(\sigma^{1}\right)\right) \\
& \leq \liminf _{k^{\prime} \rightarrow+\infty} W\left(\vec{\Xi}_{k^{\prime}} \circ \vec{\Phi}_{k^{\prime}}(\Sigma)\right) \\
& =\liminf _{k^{\prime} \rightarrow+\infty} W\left(\vec{\Phi}_{k^{\prime}}(\Sigma)\right) .
\end{aligned}
$$

Claim 1. Assume there is a node $q^{j_{1}}$ for $j_{1} \in J^{1}$ such that $\tilde{\Sigma} \cup \bigcup_{j \neq j_{1}} q^{j}$ is not disconnected. then we claim that (2.16) holds which contradict our assumption.

Proof of Claim 1. Because of (3.1) the image of the complement of $\sigma^{1}$ by $\vec{\xi}_{k}$ is connecting at the limit two distinct points of $\mathbb{R}^{m}$ images of two punctures of $\sigma^{1}$ by $\vec{\xi}_{\infty}$.

For any $\delta>0$ small enough, we cut the surface $\Sigma$ in two separated surfaces with boundaries: in one hand we consider

$$
\Sigma_{\delta}^{+}:=\phi_{k}\left(\sigma \backslash \bigcup_{j \in J^{1}} B_{\delta}^{\bar{h}}\left(q^{j}\right)\right) \subset \Sigma,
$$

and in the other hand we consider

$$
\Sigma_{\delta}^{-}:=\Sigma \backslash \Sigma_{\delta}^{+} .
$$


We split the immersions $\Xi_{k}^{1} \circ \vec{\Phi}_{k}$ according to this decomposition for some $\delta$ to be fixed later on. We observe that, since

$$
\vec{\xi}_{k} \text { converges weakly in }\left(W_{\mathrm{loc}}^{1, \infty}\left(\sigma^{1} \backslash\left[\left\{a_{1}, \ldots, a_{N}\right\} \cup\left\{q^{j}\right\}_{j \in J^{1}}\right]\right)\right)^{*},
$$

we have that

$$
\vec{\xi}_{k} \text { converges strongly in } C_{\mathrm{loc}}\left(\sigma^{1} \backslash\left[\left\{a_{1}, \ldots, a_{N}\right\} \cup\left\{q^{j}\right\}_{j \in J^{1}}\right]\right) .
$$

Hence for any $\delta>0$

$$
\forall j \in J^{1}, \quad \lim _{k \rightarrow+\infty} \sup _{2^{-1}} d\left(\vec{\xi}_{k<t<\delta}\left(\partial B_{t}^{\bar{h}}\left(q^{j}\right)\right), \vec{\xi}_{\infty}\left(\partial B_{t}^{\bar{h}}\left(q^{j}\right)\right)\right)=0
$$

where $d$ is the usual distance between two sets in $\mathbb{R}^{m}$ defined in the introduction.

Since $\tilde{h}_{k}$ is converging in $C^{\infty}$-norm to the metric $\tilde{h}$ in

$$
B_{\delta}^{\bar{h}}\left(q^{j}\right) \backslash B_{\delta / 2}^{\bar{h}}\left(q^{j}\right)
$$

which is itself conformally equivalent to the smooth constant scalar curvature met$\tilde{r}_{\tilde{h}} \bar{h}$ in $B_{\delta}^{\bar{h}}\left(q^{j}\right)$ ), we can construct conformal coordinates $\left(x_{k}^{j}, y_{k}^{j}\right)$ for the metric $\tilde{h}_{k}$ in

$$
B_{\delta}^{\bar{h}}\left(q^{j}\right) \backslash B_{\delta / 2}^{\bar{h}}\left(q^{j}\right)
$$

converging in $C^{\infty}$-norm to some limiting $\left(x_{\infty}^{j}, y_{\infty}^{j}\right)$ conformal coordinates for the metric $\tilde{h}$ (and hence also for the metric $\bar{h}$ ). Denote by $f_{k}^{j}$ the inverse of $\left(x_{k}^{j}, y_{k}^{j}\right)$ and by $f_{\infty}^{j}$ its limit. We can moreover choose $\left(x_{k}^{j}, y_{k}^{j}\right)$ in such a way that the limit $\left(x_{\infty}^{j}, y_{\infty}^{j}\right)$ goes from

$$
B_{\delta}^{\bar{h}}\left(q^{j}\right) \backslash B_{\delta / 2}^{\bar{h}}\left(q^{j}\right)
$$

into some annulus in the plane $D_{\delta}^{2} \backslash D_{c \delta}^{2}$ (note that we have $c>0$ since $\bar{h}$ is smooth around $q^{j}$ and conformally equivalent to $\left(f_{\infty}^{j}\right)^{-1}\left(d x^{2}+d y^{2}\right)$ ).

Denote $\lambda_{k}:=\log \left|\partial_{x}\left(\vec{\xi}_{k} \circ f_{k}^{j}\right)\right|=\log \left|\partial_{y}\left(\vec{\xi}_{k} \circ f_{k}^{j}\right)\right|$. The uniform bound on the area (2.3) gives that

$$
\int_{D_{\delta}^{2} \backslash D_{c \delta}^{2}} e^{2 \lambda_{k}} \leq C R^{2}
$$

Since the convergence of $\vec{\xi}_{k}$ holds true also weakly in $W_{\mathrm{loc}}^{2,2}\left(\sigma^{1} \backslash\left\{a_{1}, \ldots, a_{N}\right\} \cup\right.$ $\left.\bigcup_{j \in J}\left\{q^{j}\right\}, \bar{h}\right)$ and since (2.5) holds too, one has

$$
\lim _{k \rightarrow+\infty} \int_{D_{\delta}^{2} \backslash D_{c \delta}^{2}}\left|\lambda_{k}-\lambda_{\infty}\right|^{2}=0 .
$$


The combination of (3.3) and (3.4) gives that

$$
\lim _{k \rightarrow+\infty} \int_{D_{\delta}^{2} \backslash D_{c \delta}^{2}}\left|e^{\lambda_{k}}-e^{\lambda_{\infty}}\right|=0 .
$$

Hence there exists $\alpha_{k} \in(c, 1)$ such that

$$
\lim _{k \rightarrow+\infty} \int_{\partial D_{\alpha_{k} \delta}^{2}}\left|e^{\lambda_{k}}-e^{\lambda \infty}\right|=0 .
$$

Since $\vec{\xi}_{\infty} \in \mathcal{E}_{\Sigma}, \vec{\xi}_{\infty} \circ f_{\infty}^{j} \in W^{1, \infty}\left(D^{2}\right)$ and $e^{\lambda \infty} \in L^{\infty}\left(D^{2}\right)$. Thus

$$
\int_{D_{\alpha_{k} \delta}^{2}} e^{\lambda \infty}=O(\delta)
$$

Combining (3.6) and (3.7), we obtain that there exists $C>0$ such that for any $j \in J$ and for any $\delta>0$ there exists $k_{\delta} \in \mathbb{N}$ verifying

$$
\forall k \geq k_{\delta} \exists \alpha_{k}^{j} \in(1 / 2,1): \quad \mathscr{H}^{1}\left(\vec{\xi}_{k}\left(\partial B_{\alpha_{k}^{j} \delta}^{\bar{h}}\right)\right) \leq C \delta .
$$

Since there is a node $q^{j_{1}}$ for $j_{1} \in J^{1}$ such that $\tilde{\Sigma} \cup \bigcup_{j \neq j_{1}} q^{j}$ is not disconnected and since (3.1) holds, there must exist a sequence of points $p_{k} \in \Sigma_{\delta / 2}^{-}$such that

$$
\liminf _{k \rightarrow+\infty} \inf _{j \in J^{1}}\left|p_{k}-q^{j}\right|>0
$$

Cut now $\Sigma$ into at least two disjoint connected components by removing the curves $\partial B_{\alpha_{k}^{j} \delta}^{\bar{h}}$. Applying twice the monotonicity formula with boundary Lemma A.3 with

$$
T \rightarrow+\infty, \quad t \rightarrow 0
$$

and with respect respectively to an arbitrary fixed point $p \in \Sigma_{\delta / 2}^{+}$for the immersion $\vec{\xi}_{k}$ restricted to $\Sigma_{\delta / 2}^{+}$and with respect to $p_{k}$ for the immersion $\vec{\xi}_{k}$ restricted to $\Sigma_{\delta / 2}^{-}$one obtains

$$
\begin{aligned}
\liminf _{k \rightarrow+\infty} W\left(\vec{\Phi}_{k}\right) & =\liminf _{k \rightarrow+\infty} W\left(\Xi_{k}^{1} \circ \vec{\Phi}_{k}\right) \\
& \geq \liminf _{k \rightarrow+\infty} W\left(\vec{\xi}_{k}\left(\Sigma_{\delta}^{+}\right)\right)+W\left(\vec{\xi}_{k}\left(\Sigma_{\delta}^{-}\right)\right) \\
& \geq 4 \pi+4 \pi-C \delta=8 \pi-C \delta .
\end{aligned}
$$

Since this holds for any $\delta>0$, we have then (2.16) which contradicts our assumption and we have proved the claim. 
Denote by $g\left(\sigma^{i}\right)$ the genus of each component $\sigma^{i}$ of the surface $\tilde{\Sigma}$. If each $q^{j}$ for $j=1, \ldots, n$ is disconnecting $\tilde{\Sigma}$, the Deligne-Mumford's description of the loss of compactness in the Moduli space gives

$$
g(\Sigma)=\sum_{j=1}^{M} g\left(\sigma^{j}\right) .
$$

Starting now from this normalized sequence $\Xi_{k}^{1} \circ \Phi_{k}$ for the first component $\sigma^{1}$, because of the convergences (2.4) and (2.5) we have

$$
\lim _{\delta \rightarrow 0} \liminf _{k \rightarrow+\infty} W\left(\vec{\xi}_{k}\left(\sigma_{\delta}^{1}\right)\right) \geq W\left(\vec{\xi}_{\infty}\left(\sigma^{1}\right)\right) \geq \beta_{g\left(\sigma^{1}\right)} .
$$

For each of the other components $\sigma^{i}, i \neq 1$, working under the condition that (2.16) does not hold, Proposition 2.1 gives for each $i$ the existence of a subsequence - still denoted by $\xi_{k}$ - and the existence of a sequence of Möbius transformations such that $\vec{\Xi}_{k}^{i} \circ \vec{\Xi}_{k}^{1} \circ \vec{\Phi}_{k}$ converges in the sense (2.4) and (2.5). Taking subsequences of subsequences, we can assume that the subsequence we are working with is common to all the $\sigma^{i}$. As for $\sigma^{1}$ we have for any $i$

$$
\lim _{\delta \rightarrow 0} \liminf _{k \rightarrow+\infty} W\left(\Xi_{k}^{i} \circ \vec{\xi}_{k}\left(\sigma_{\delta}^{i}\right)\right) \geq W\left(\vec{\xi}_{i}^{\infty}\right) \geq \beta_{g\left(\sigma^{i}\right)},
$$

where $\vec{\xi}_{\infty}^{i}$ is the weak local limit of $\Xi_{k}^{i} \circ \vec{\xi}_{k}$ on $\sigma^{i}$ minus the blow up points and the punctures. Using now Lemma A.4, we deduce that for any $i \neq 1$

$$
\lim _{\delta \rightarrow 0} \liminf _{k \rightarrow+\infty} W\left(\vec{\xi}_{k}\left(\sigma_{\delta}^{i}\right)\right) \geq W\left(\vec{\xi}_{i}^{\infty}\right)-4 \pi \geq \beta_{g\left(\sigma^{i}\right)}-4 \pi .
$$

Summing over $i$ gives that

$$
\begin{aligned}
\liminf _{k \rightarrow+\infty} W\left(\vec{\Phi}_{k}\right) & =\liminf _{k \rightarrow+\infty} W\left(\Xi_{k}^{1} \circ \vec{\Phi}_{k}\right) \\
& \geq \lim _{\delta \rightarrow 0} \liminf _{k \rightarrow+\infty} \sum_{i=1}^{M} W\left(\vec{\xi}_{k}\left(\sigma^{i}\right)\right) \geq \beta_{g\left(\sigma^{1}\right)}+\sum_{i=1}^{M}\left(\beta_{g\left(\sigma^{i}\right)}-4 \pi\right) .
\end{aligned}
$$

This concludes the proof of Theorem 1.1 for $g>1$.

Proof of Theorem 1.1 for $g=1$. The degenerating torus has a conformal class close to the flat torus $[0,1] \times[-k, k]$ and we can restrict to this case in order to simplify the notations. We fix three distinct points $P_{1}:=(0,0), P_{2}:=(0,1)$ and $P_{3}:=(0,-1)$ and we apply the Three Point Normalization Lemma A.1. 
If the resulting conformal immersion $\vec{\xi}_{k}$ would degenerate in any compact subset $[0,1] \times[-A, A]$,

$$
\log \left|d \vec{\xi}_{k}\right| \rightarrow-\infty \quad \text { uniformly on }[0,1] \times[-A, A] \backslash\left\{a_{1}, \ldots, a_{N}\right\}
$$

where $a_{i}$ are the concentration points for the energy $\int\left|\nabla \vec{n}_{\vec{\xi}_{k}}\right|^{2} d x d y$, then arguing exactly as in the proof of Claim 2 in Section 2 we would have inequality (2.1) and the theorem would be proved for $g=1$. Therefore we only have to consider the case when $\vec{\xi}_{k}$ converges weakly in $W_{\text {loc }}^{2,2}$ to a non trivial conformal immersion $\vec{\xi}_{\infty}$ on any annulus $[0,1] \times[-A, A]$ away from the blow up points $\left\{a_{1}, \ldots, a_{N}\right\}$.

Since

$$
\limsup _{A \rightarrow+\infty} \int_{[0,1] \times[-A, A]}\left|\nabla \vec{n}_{\vec{\xi}_{\infty}}\right|^{2} d x d y<+\infty,
$$

it follows that, by using for instance [13, Lemma A.5], $\vec{\xi}_{\infty}$ extends to a possibly branched conformal immersion of the sphere $S^{2} \simeq[0,1] \times[-\infty,+\infty]$ where the North and the South poles can be seen as two punctures $q^{1}$ and $q^{2}$ in the DeligneMumford compactification framework described in Section 2.

If $\vec{\xi}_{\infty}$ (North) $=\vec{\xi}_{\infty}$ (South), then we can argue exactly as in the end of Section 2 and we would have

$$
\begin{aligned}
\liminf _{k^{\prime} \rightarrow+\infty} W\left(\vec{\Phi}_{k^{\prime}}(\Sigma)\right) & =\liminf _{k^{\prime} \rightarrow+\infty} W\left(\vec{\Xi}_{k^{\prime}} \circ \vec{\Phi}_{k^{\prime}}(\Sigma)\right) \\
& \geq \liminf _{k^{\prime} \rightarrow+\infty} W\left(\vec{\xi}_{k^{\prime}}([0,1] \times[-A, A])\right) \\
& \geq W\left(\vec{\xi}_{\infty}\left(S^{2}\right)\right) \geq 8 \pi
\end{aligned}
$$

which would again conclude the proof of Theorem 1.1 for $g=1$.

Hence we are now restricting to the case $\vec{\xi}_{\infty}$ (North) $\neq \vec{\xi}_{\infty}$ (South) and splitting the torus $[0,1] \times[-k, k]$ into two connected components by cutting it along the circles $[0,1] \times\{-A\}$ and $[0,1] \times\{A\}$ we can argue exactly as in the proof of Claim 1 of the present Section 3: we can make use of the monotonicity formula with boundary Lemma A.3 and deduce

$$
\begin{aligned}
\liminf _{k \rightarrow+\infty} W\left(\vec{\Phi}_{k}\right)= & \liminf _{k \rightarrow+\infty} W\left(\Xi_{k}^{1} \circ \vec{\Phi}_{k}\right) \\
\geq & \liminf _{k \rightarrow+\infty}\left(W\left(\vec{\xi}_{k}([0,1] \times[-A, A])\right)\right. \\
& \left.\quad+W\left(\vec{\xi}_{k}([0,1] \times[-k,-A] \cup[0,1] \times[A, k])\right)\right) \\
\geq & 4 \pi+4 \pi-o_{A}(1)=8 \pi-o_{A}(1) .
\end{aligned}
$$

where $o_{A}(1)$ converges to zero as $A$ goes to $+\infty$. This concludes the proof of Theorem 1.1 for $g=1$. 


\section{A Appendix}

Lemma A.1 (Three Points Normalization Lemma). For any $\Lambda>0$ there exists $R, r>0$ such that for any closed 2-dimensional manifold $\Sigma$, for any choice of three distinct points $P_{1}, P_{2}$ and $P_{3}$ in $\Sigma$ and for any embedding $\vec{\Phi}$ of $\Sigma$ into $\mathbb{R}^{m}$ satisfying

$$
\int_{\Sigma}\left|d \vec{n}_{\vec{\Phi}}\right|_{g}^{2} d \operatorname{vol}_{g}<\Lambda
$$

where $g:=\vec{\Phi}^{*} g_{\mathbb{R}^{m}}$, then there exists a Möbius transformation $\Xi$ of $\mathbb{R}^{m}$ such that

$$
\Xi \circ \vec{\Phi}(\Sigma) \subset B_{R}(0) \quad \text { and } \quad \forall i \neq j, \quad\left|\Xi \circ \vec{\Phi}\left(P_{i}\right)-\Xi \circ \vec{\Phi}\left(P_{j}\right)\right| \geq r .
$$

Moreover the following control of the total area of $\Xi \circ \vec{\Phi}(\Sigma)$ holds:

$$
\mathscr{H}^{2}(\Xi \circ \vec{\Phi}(\Sigma)) \leq C R^{2} \Lambda
$$

where $C>0$ is a universal constant.

Proof. We apply a translation and a dilation in such a way that $P_{1}=0$ and

$$
\left|\vec{\Phi}\left(P_{1}\right)-\vec{\Phi}\left(P_{2}\right)\right|=\min _{i \neq j}\left|\vec{\Phi}\left(P_{i}\right)-\vec{\Phi}\left(P_{j}\right)\right|=1 .
$$

We keep denoting $\vec{\Phi}$ the resulting embedding - observe that due to its conformal invariance the Willmore energy has not been modified. From Lemma A.3 there exists a universal constant $C>0$ such that for any $x_{0} \in \mathbb{R}^{m}$ and $0<\sigma<\rho<+\infty$

$$
\begin{aligned}
\sigma^{-2} \mathcal{H}^{2}\left(\vec{\Phi}(\Sigma) \cap B_{\sigma}\left(x_{0}\right)\right) \leq C & {\left[\rho^{-2} \mathcal{H}^{2}\left(\vec{\Phi}(\Sigma) \cap B_{\rho}\left(x_{0}\right)\right)\right.} \\
& \left.+\int_{\vec{\Phi}^{-1}\left(B_{\rho}\left(x_{0}\right)\right)}|\vec{H}|^{2} d \operatorname{vol}_{g}\right] .
\end{aligned}
$$

We claim that there exists $\rho_{0}$ depending only on $\Lambda>W(\vec{\Phi})$ and $x_{1} \in B_{1}(0)$ such that $\vec{\Phi}(\Sigma) \cap B_{\rho_{0}}\left(x_{1}\right)=\emptyset$. For $y \in \vec{\Phi}(\Sigma)$ one has

$$
\lim _{\sigma \rightarrow 0} \sigma^{-2} \mathscr{H}^{2}\left(\vec{\Phi}(\Sigma) \cap B_{\sigma}(y)\right)=\pi .
$$

For $0<\rho<1 / 2$ we consider a regular covering of $B_{1}(0)$ by balls $B_{\rho}\left(z_{l}\right)$ in such a way that any point in $B_{1}(0)$ is contained in at most $C(m)$ balls of the form $B_{2 \rho}\left(z_{l}\right)$. The number of $l$ such that

$$
\int_{\vec{\Phi}^{-1}\left(B_{2 \rho}\left(z_{l}\right)\right)}|\vec{H}|^{2} d \operatorname{vol}_{g}>C^{-1} \frac{\pi}{2}
$$


is bounded by $2 \Lambda C C(m)$. For an $l$ such that

$$
\int_{\vec{\Phi}^{-1}\left(B_{2 \rho}\left(z_{l}\right)\right)}|\vec{H}|^{2} d \operatorname{vol}_{g}<C^{-1} \frac{\pi}{2}
$$

and such that there exists $y \in B_{\rho}\left(z_{l}\right) \cap \Sigma \neq \emptyset$, combining (A.4) and (A.5) one obtains that

$$
(2 \rho)^{-2} \mathscr{H}^{2}\left(\vec{\Phi}(\Sigma) \cap B_{2 \rho}\left(z_{l}\right)\right)>C^{-1} \frac{\pi}{2} .
$$

the number of such $l$ is then bounded by $\rho^{-2}$ times a number depending only on $m$ and $\Lambda$ - where we are using again (A.4) but for $x_{0}=0, \sigma=1$ and $\rho \rightarrow+\infty$. The total number of ball $B_{\rho}\left(z_{l}\right)$ is proportional to $\rho^{-m}$. Since $m>2$, for $\rho=\rho_{0}$ chosen small enough, depending only on $m$ and $\Lambda$ we deduce the claim.

Let $x_{1}$ and $\rho_{0}$ given by the claim we choose $\Xi$ to be the inversion with respect to $x_{1}$ :

$$
\Xi(x):=\frac{x-x_{1}}{\left|x-x_{1}\right|^{2}} .
$$

We have then

$$
\Xi(\vec{\Phi}(\Sigma)) \subset B_{1 / \rho_{0}}(0),
$$

moreover, since none of the $P_{i}$ is in $B_{\rho_{0}}\left(x_{1}\right)$, we have that

$$
\forall i=1,2,3, \quad \Xi\left(\vec{\Phi}\left(P_{i}\right)\right) \in B_{1 / \rho_{0}}(0) .
$$

We have also that $\left|\vec{\Phi}\left(P_{2}\right)-x_{1}\right|+\left|\vec{\Phi}\left(P_{1}\right)-x_{1}\right|<3$ hence $\Xi\left(\vec{\Phi}\left(P_{1}\right)\right)$ and $\Xi\left(\vec{\Phi}\left(P_{2}\right)\right)$ are contained in $\mathbb{R}^{m} \backslash B_{1 / 3}(0)$, thus

$\left|\Xi\left(\vec{\Phi}\left(P_{1}\right)\right)-\Xi\left(\vec{\Phi}\left(P_{2}\right)\right)\right|\left\|\nabla \Xi^{-1}\right\|_{L^{\infty}\left(B_{1 / \rho_{0}}(0) \backslash B_{1 / 3}(0)\right)} \geq\left|\vec{\Phi}\left(P_{1}\right)-\vec{\Phi}\left(P_{2}\right)\right|=1$,

which implies that

$$
\left|\Xi\left(\vec{\Phi}\left(P_{1}\right)\right)-\Xi\left(\vec{\Phi}\left(P_{2}\right)\right)\right| \geq 9 .
$$

Either $\vec{\Phi}\left(P_{3}\right) \in B_{10}(0)$ or $\vec{\Phi}\left(P_{3}\right) \in \mathbb{R}^{m} \backslash B_{10}(0)$. In the first case one has that all the $\Xi\left(\vec{\Phi}\left(P_{i}\right)\right)$ are included in $B_{1 / \rho_{0}}(0) \backslash B_{1 / 11}(0)$, hence we have

$$
\begin{gathered}
\forall i \neq j, \quad\left|\Xi\left(\vec{\Phi}\left(P_{i}\right)\right)-\Xi\left(\vec{\Phi}\left(P_{j}\right)\right)\right|\left\|\nabla \Xi^{-1}\right\|_{L^{\infty}\left(B_{1 / \rho_{0}}(0) \backslash B_{1 / 11}(0)\right)} \\
\geq\left|\vec{\Phi}\left(P_{i}\right)-\vec{\Phi}\left(P_{j}\right)\right| \geq 1
\end{gathered}
$$

which implies that

$$
\forall i \neq j, \quad\left|\Xi\left(\vec{\Phi}\left(P_{i}\right)\right)-\Xi\left(\vec{\Phi}\left(P_{j}\right)\right)\right| \geq 11^{2} .
$$

This implies (A.2) in this case. In the case when $\vec{\Phi}\left(P_{3}\right) \in \mathbb{R}^{m} \backslash B_{10}(0)$, we deduce that $\Xi\left(\vec{\Phi}\left(P_{3}\right)\right) \in B_{1 / 9}(0)$ and since $\Xi\left(\vec{\Phi}\left(P_{1}\right)\right)$ and $\Xi\left(\vec{\Phi}\left(P_{2}\right)\right)$ are contained in 
$\mathbb{R}^{m} \backslash B_{1 / 3}(0)$, we obtain that

$$
\forall i=1,2, \quad\left|\Xi\left(\vec{\Phi}\left(P_{i}\right)\right)-\Xi\left(\vec{\Phi}\left(P_{3}\right)\right)\right| \geq \frac{2}{9} .
$$

This lower bound combined with (A.7) gives (A.2) in this case too.

Regarding the proof of estimate (A.3), we first observe that inequality (A.4) (which holds also for $\vec{\Phi}$ replaced by $\Xi \circ \vec{\Phi}$ ) implies that, for any $\rho \geq \rho_{0}^{-1}$

$$
\mathscr{H}^{2}(\Xi \circ \vec{\Phi}(\Sigma)) \leq C \rho_{0}^{-2} \rho^{-2} \mathscr{H}^{2}(\Xi \circ \vec{\Phi}(\Sigma))+\rho_{0}^{-2} \Lambda \text {. }
$$

Letting $\rho$ converge to $+\infty$ yields the desired estimate (A.3). Hence Lemma A.1 is proved.

The following lemma is more or less implicitly contained in [10] and [4]. We prove it however for the convenience of the reader.

Lemma A.2. Let $\vec{\Phi}_{k}$ be a sequence of conformal immersions of the disc $D^{2}$ into $\mathbb{R}^{m}$ such that

$$
\sup _{k \in \mathbb{N}} \int_{D^{2}}\left|\nabla \vec{n}_{\vec{\Phi}_{k}}\right|^{2} d x d y \leq \frac{8 \pi}{3}
$$

and

$$
\limsup _{k \rightarrow+\infty} \int_{D^{2}} e^{2 \lambda_{k}} d x d y<+\infty
$$

where $e^{\lambda_{k}}=\left|\partial_{x} \vec{\Phi}_{k}\right|=\left|\partial_{y} \vec{\Phi}_{k}\right|$. Then the following alternative holds: either

$$
\forall \omega \subset \subset D^{2}, \quad \lim _{k \rightarrow+\infty} \lambda_{k}=-\infty \quad \text { uniformly on } \omega
$$

or there exists a subsequence $k^{\prime}$ such that

$$
\forall \omega \subset \subset D^{2}, \quad \limsup _{k^{\prime} \rightarrow+\infty}\left\|\lambda_{k^{\prime}}\right\|_{L^{\infty}(\omega)}<+\infty .
$$

Proof. Since (A.10) holds, [4, Lemma 5.1.4] gives the existence of a moving frame $\left(\vec{e}_{1}, \vec{e}_{2}\right) \in\left(W^{1,2}\left(D^{2}, S^{m-1}\right)\right)^{2}$ such that

$$
\int_{D^{2}}\left|\nabla \vec{e}_{1}\right|^{2}+\left|\nabla \vec{e}_{2}\right|^{2} \leq C \int_{D^{2}}\left|\nabla \vec{n}_{\vec{\Phi}_{k}}\right|^{2} d x d y \leq \frac{8 \pi}{3},
$$

where $C>0$ only depends on $m$ and

$$
\star \vec{n}_{\vec{\Phi}}=\vec{e}_{1} \wedge \vec{e}_{2}
$$

Moreover $\lambda_{k}$ satisfies

$$
\Delta \lambda_{k}=\left(\nabla^{\perp} \vec{e}_{1}, \nabla \vec{e}_{2}\right) .
$$


Let $\mu_{k}$ be the solution of

$$
\left\{\begin{aligned}
\Delta \mu_{k} & =\left(\nabla^{\perp} \vec{e}_{1}, \nabla \vec{e}_{2}\right) & & \text { in } D^{2}, \\
\mu_{k} & =0 & & \text { on } \partial D^{2} .
\end{aligned}\right.
$$

Wente's theorem (see [4, Theorems 3.1.2 and 3.1.9]) asserts that

$$
\begin{aligned}
\left\|\mu_{k}\right\|_{L^{\infty}\left(D^{2}\right)} & \leq(2 \pi)^{-1}\left\|\nabla \vec{e}_{1}\right\|_{L^{2}}\left\|\nabla \vec{e}_{2}\right\|_{L^{2}} \\
& \leq \pi^{-1} C \int_{D^{2}}\left|\nabla \vec{n}_{\vec{\Phi}_{k}}\right|^{2} d x d y .
\end{aligned}
$$

Hence $\mu_{k}$ is uniformly bounded in $L^{\infty}$-norm on $D^{2}$.

The function $v_{k}:=\lambda_{k}-\mu_{k}$ is harmonic on $D^{2}$ and satisfies

$$
\limsup _{k \rightarrow+\infty} \int_{D^{2}} e^{2 v_{k}} d x d y<+\infty \text {. }
$$

Let $\omega$ be an open set strictly included in $D^{2}$ (i.e. $\bar{\omega} \subset D^{2}$ ) and let $U$ be an open set strictly included in $D^{2}$ and such that $\omega$ is itself strictly included in $U$, that is, $\omega \subset \subset U \subset \subset D^{2}$. Since $e^{2 v_{k}}$ is subharmonic $\left(\Delta e^{2 v_{k}} \geq 0\right)$ and positive, from Harnack's inequality there exists a constant $C$ depending on $U$ such that

$$
v_{k}^{+}=\sup _{U} v_{k} \leq \log \left[C \int_{D^{2}} e^{2 v_{k}}\right]
$$

So $v_{k}^{+}$is uniformly bounded from above. If

$$
v_{k}^{+} \rightarrow-\infty
$$

then the first alternative in the lemma holds for this special $\omega$. Assuming on the contrary that

$$
\lim \sup v_{k}^{+}>-\infty,
$$

there exists a subsequence $k^{\prime}$ such that

$$
v_{k^{\prime}}^{+} \rightarrow v_{\infty}^{+} \in \mathbb{R} .
$$

Consider now the sequence of positive harmonic functions $v_{k^{\prime}}^{+}-v_{k^{\prime}}$ on $U$, Harnack's inequality again gives the existence of a constant $C>0$ independent of $k^{\prime}$ such that

$$
\sup _{\omega} v_{k^{\prime}}^{+}-v_{k^{\prime}} \leq C \inf _{\omega} v_{k^{\prime}}^{+}-v_{k^{\prime}}
$$

It is clear also that the supremum of $v_{k^{\prime}}$ is bounded from above on $\omega$, therefore

$$
\limsup _{k^{\prime} \rightarrow+\infty} \inf _{\omega} v_{k^{\prime}}^{+}-v_{k^{\prime}}<+\infty \text {. }
$$


Combining the two last inequalities gives that

$$
\liminf \inf _{\omega} v_{k^{\prime}}>+\infty \text {. }
$$

Thus the second alternative of the lemma holds for this special $\omega$.

So we have proved that for any strict sub-domain of $D^{2}$ one of the two alternatives always holds. Now it is clear that if for some sub-domain of $D^{2}$ the first alternative holds, then the second cannot hold for another sub-domain and vice versa. Thus Lemma A.2 is proved.

The following lemma is the extension of Simon's monotonicity formula ${ }^{4}$ in the presence of a boundary. This lemma might have been already published somewhere, but the author could not find it anywhere and is making the computation related to it available to the reader.

Lemma A.3 (Monotonicity formula with boundary). Let $\Sigma$ be a smooth compact surface with boundary and let $\vec{\Phi}$ be an element of $\mathcal{E}_{\Sigma}$, a Lipschitz immersion of the surface $\Sigma$ into $\mathbb{R}^{m}$ with $L^{2}$ bounded second fundamental form. Denote by $M:=\vec{\Phi}(\Sigma)$ the immersed surface. Then for any point $\vec{x}^{0} \in \mathbb{R}^{m}$ and any choice of two radii $0<t<T<+\infty$ the following identity holds:

$$
\begin{aligned}
T^{-2} \operatorname{Area}\left(M \cap B_{T}\left(\vec{x}^{0}\right)\right)-t^{-2} \operatorname{Area}\left(M \cap B_{t}\left(\vec{x}^{0}\right)\right) \\
=\int_{M \cap B_{T}\left(\vec{x}^{0}\right) \backslash B_{t}\left(\vec{x}^{0}\right)}\left|\frac{\left(\vec{x}-\vec{x}^{0}\right)^{\perp}}{\left|\vec{x}-\vec{x}^{0}\right|^{2}}+\frac{\vec{H}}{2}\right|^{2} d \operatorname{vol}_{g} \\
\quad-\frac{1}{4} \int_{M \cap B_{T}\left(\vec{x}^{0}\right) \backslash B_{t}\left(\vec{x}^{0}\right)}|\vec{H}|^{2} d \operatorname{vol}_{g} \\
\quad-\frac{1}{T^{2}} \int_{M \cap B_{T}\left(\vec{x}^{0}\right)}\left\langle\vec{x}-\vec{x}^{0}, \vec{H}\right\rangle d \operatorname{vol}_{g} \\
+\frac{1}{t^{2}} \int_{M \cap B_{t}\left(\vec{x}^{0}\right)}\left\langle\vec{x}-\vec{x}^{0}, \vec{H}\right\rangle d \operatorname{vol}_{g} \\
+\frac{1}{2} \int_{\partial M \cap B_{T}\left(\vec{x}^{0}\right)}\left(\frac{1}{T^{2}}-\frac{1}{\rho_{t}^{2}}\right)\left\langle\vec{x}-\vec{x}^{0}, \vec{v}\right\rangle d l_{\partial M}
\end{aligned}
$$

where $\left(\vec{x}-\vec{x}^{0}\right)^{\perp}$ is the orthogonal projection of the vector $\vec{x}-\vec{x}^{0}$ onto $\left(T_{\vec{x}} M\right)^{\perp}$ the normal plane to the surface at $\vec{x}$ and $\rho_{t}:=\max \left\{\left|\vec{x}-\vec{x}^{0}\right|, t\right\}$.

Proof. To simplify the presentation we give the argument for $\Sigma=D^{2}$. We follow step by step the computations in [14, pp. 82-84].

${ }^{4}$ Formula (A.13) is exactly the formula as it is written in [8, formula (A.3), p. 353] except that the boundary term was not considered in this work. 
Let $\vec{X}$ be a smooth vector-field in $\mathbb{R}^{m}$. We define the divergence of $\vec{X}$ along $M:=\vec{\Phi}\left(D^{2}\right)$ to be the following quantity:

$$
\operatorname{div}_{M} \vec{X}:=\sum_{k=1}^{2}\left\langle d \vec{X} \cdot \vec{e}_{k}, \vec{e}_{k}\right\rangle
$$

where $\left(\vec{e}_{1}, \vec{e}_{2}\right)$ is an arbitrary local orthonormal frame on $M$. Decomposing $\vec{X}$ along $M$ in the sum of its tangential

$$
\vec{X}^{T}:=\sum_{k=1}^{2}\left\langle\vec{X}, \vec{e}_{k}\right\rangle \vec{e}_{k}
$$

and its vertical part

$$
\vec{X}^{\perp}=\vec{X}-\vec{X}^{T}
$$

gives $^{5}$

$$
\begin{aligned}
\operatorname{div}_{M} \vec{X} & =\operatorname{div}_{M} \vec{X}^{T}+\operatorname{div}_{M} \vec{X}^{\perp} \\
& =\sum_{k=1}^{2}\left\langle d \vec{X}^{T} \cdot \vec{e}_{k}, \vec{e}_{k}\right\rangle-\left\langle\vec{X}^{\perp}, \sum_{k=1}^{2} d \vec{e}_{k} \cdot \vec{e}_{k}\right\rangle \\
& =\sum_{k=1}^{2}\left\langle d \vec{X}^{T} \cdot \vec{e}_{k}, \vec{e}_{k}\right\rangle-2\langle\vec{X}, \vec{H}\rangle .
\end{aligned}
$$

We can assume that $\vec{\Phi}$ is conformal since such a conformal reparametrization always exists (see [11]). Denote $e^{\lambda}:=\left|\partial_{x_{1}} \vec{\Phi}\right|=\left|\partial_{x_{2}} \vec{\Phi}\right|$ and take $\vec{e}_{k}:=e^{-\lambda} \partial_{x_{k}} \vec{\Phi}$. Write $\vec{X}^{T}:=\sum_{i=1}^{2} X_{i} \partial_{x_{i}} \vec{\Phi}$. We have

$$
\operatorname{div}_{M} \vec{X}^{T}=\sum_{k=1}^{2}\left\langle d \vec{X} \cdot \vec{e}_{k}, \vec{e}_{k}\right\rangle=\sum_{i=1}^{2} \frac{\partial X_{i}}{\partial x_{i}}+\sum_{i=1}^{2} X_{i} e^{-2 \lambda} \partial_{x_{i}} e^{2 \lambda}
$$

Hence

$$
\begin{aligned}
\int_{M} \operatorname{div}_{M} \vec{X}^{T} d \operatorname{vol}_{g} & =\int_{D^{2}} \sum_{i=1}^{2} \frac{\partial}{\partial x_{i}}\left(e^{2 \lambda} X_{i}\right) d x_{1} d x_{2} \\
& =\int_{0}^{2 \pi} e^{2 \lambda} \sum_{i=1}^{2} X_{i} x_{i} d \theta \\
& =\int_{\partial M}\left\langle\vec{X}^{T}, \vec{v}\right\rangle d l_{\partial M}=\int_{\partial M}\langle\vec{X}, \vec{v}\rangle d l_{\partial M}
\end{aligned}
$$

${ }^{5}$ In comparison with formula in [14] observe that our definition of the mean curvature vector differs by a factor 2 . 
where $\vec{v}$ is the unit limiting tangent vector to $M$ on $\partial M$ orthogonal to it and oriented in the outward direction, i.e. $\vec{v}:=e^{-\lambda} \partial_{r} \vec{\Phi}$. Combining (A.15) and (A.17) gives then

$$
\int_{M} \operatorname{div}_{M} \vec{X} d \operatorname{vol}_{g}=\int_{\partial M}\langle\vec{X}, \vec{v}\rangle d l_{\partial M}-2 \int_{M}\langle\vec{X}, \vec{H}\rangle d \operatorname{vol}_{g} .
$$

As in [14] we choose $\vec{X}:=\gamma(\rho)\left(\vec{x}-\vec{x}^{0}\right)$ where $\vec{x}^{0}$ is an arbitrary point in $\mathbb{R}^{m}$ and $\rho=\left|\vec{x}-\vec{x}^{0}\right|$. We have for this choice of $\vec{X}$

$$
\begin{aligned}
\operatorname{div}_{M} \vec{X} & =2 \gamma+\dot{\gamma} \sum_{k=1}^{2} d r \cdot \vec{e}_{k}\left\langle\vec{x}-\vec{x}^{0}, \vec{e}_{k}\right\rangle \\
& =2 \gamma+\rho \dot{\gamma}\left[1-\frac{\left|\left(\vec{x}-\vec{x}^{0}\right)^{\perp}\right|^{2}}{\left|\vec{x}-\vec{x}^{0}\right|^{2}}\right] .
\end{aligned}
$$

We choose now $\gamma(\rho)$ to be a function depending on a parameter $s>0$, that is, $\gamma_{s}(\rho)=\varphi(\rho / s)$ where later on $\varphi$ will be chosen to be closer and closer to the characteristic function of the unit interval $[0,1]$. Then (A.18) becomes

$$
\begin{gathered}
2 \int_{M} \varphi\left(\frac{\rho}{s}\right) d \operatorname{vol}_{g}+\int_{M} \dot{\varphi}\left(\frac{\rho}{s}\right) \frac{\rho}{s}\left[1-\frac{\left|\left(\vec{x}-\vec{x}^{0}\right)^{\perp}\right|^{2}}{\left|\vec{x}-\vec{x}^{0}\right|^{2}}\right] d \operatorname{vol}_{g} \\
=\int_{\partial M} \varphi\left(\frac{\rho}{s}\right)\left\langle\vec{x}-\vec{x}^{0}, \vec{v}\right\rangle d l_{\partial M} \\
\quad-2 \int_{M} \varphi\left(\frac{\rho}{S}\right)\left\langle\vec{x}-\vec{x}^{0}, \vec{H}\right\rangle d \operatorname{vol}_{g} .
\end{gathered}
$$

Observe that

$$
\frac{d}{d s}\left[\varphi\left(\frac{\rho}{s}\right) \frac{1}{s^{2}}\right]=-\frac{1}{s^{3}}\left[2 \varphi\left(\frac{\rho}{s}\right)+\dot{\varphi}\left(\frac{\rho}{s}\right) \frac{\rho}{s}\right]
$$

Hence we deduce that

$$
\begin{aligned}
-\frac{d}{d s}\left[\frac{1}{s^{2}} \int_{M} \varphi\left(\frac{\rho}{s}\right) d \operatorname{vol}_{g}\right] & \\
=-\frac{1}{s^{2}} \frac{d}{d s} & {\left[\int_{M} \varphi\left(\frac{\rho}{s}\right) \frac{\left|\left(\vec{x}-\vec{x}^{0}\right)^{\perp}\right|^{2}}{\left|\vec{x}-\vec{x}^{0}\right|^{2}} d \operatorname{vol}_{g}\right] } \\
& +\frac{1}{s^{3}} \int_{\partial M} \varphi\left(\frac{\rho}{s}\right)\left\langle\vec{x}-\vec{x}^{0}, \vec{v}\right\rangle d l_{\partial M} \\
& -\frac{2}{s^{3}} \int_{M} \varphi\left(\frac{\rho}{s}\right)\left\langle\vec{x}-\vec{x}^{0}, \vec{H}\right\rangle d \operatorname{vol}_{g}
\end{aligned}
$$


Taking $\varphi$ closer and closer to the characteristic function of the unit interval $[0,1]$, (A.20) implies at the limit the following formula: ${ }^{6}$

$$
\begin{aligned}
\frac{d}{d s}\left[\frac{1}{s^{2}} \int_{M \cap B_{s}\left(\vec{x}^{0}\right)} d \operatorname{vol}_{g}\right]=\frac{d}{d s} & {\left[\int_{M \cap B_{s}\left(\vec{x}^{0}\right)} \frac{\left|\left(\vec{x}-\vec{x}^{0}\right)^{\perp}\right|^{2}}{\left|\vec{x}-\vec{x}^{0}\right|^{4}} d \operatorname{vol}_{g}\right] } \\
& -\frac{1}{s^{3}} \int_{\partial M \cap B_{S}\left(\vec{x}^{0}\right)}\left\langle\vec{x}-\vec{x}^{0}, \vec{v}\right\rangle d l_{\partial M} \\
& +\frac{2}{s^{3}} \int_{M \cap B_{S}\left(\vec{x}^{0}\right)}\left\langle\vec{x}-\vec{x}^{0}, \vec{H}\right\rangle d \operatorname{vol}_{g} .
\end{aligned}
$$

Integrating this formula between $0<t<T<+\infty$ gives

$$
\begin{aligned}
& T^{-2} \operatorname{Area}\left(M \cap B_{T}\left(\vec{x}^{0}\right)\right)-t^{-2} \operatorname{Area}\left(M \cap B_{t}\left(\vec{x}^{0}\right)\right) \\
& =\int_{M \cap B_{T}\left(\vec{x}^{0}\right) \backslash B_{t}\left(\vec{x}^{0}\right)} \frac{\left|\left(\vec{x}-\vec{x}^{0}\right)^{\perp}\right|^{2}}{\left|\vec{x}-\vec{x}^{0}\right|^{4}} d \operatorname{vol}_{g} \\
& \quad+\frac{1}{2} \int_{\partial M \cap B_{T}\left(\vec{x}^{0}\right)}\left(\frac{1}{T^{2}}-\frac{1}{\rho_{t}^{2}}\right)\left\langle\vec{x}-\vec{x}^{0}, \vec{v}\right\rangle d l_{\partial M} \\
& \quad+\int_{M \cap B_{T}\left(\vec{x}^{0}\right)}\left(\frac{1}{\rho_{t}^{2}}-\frac{1}{T^{2}}\right)\left\langle\vec{x}-\vec{x}^{0}, \vec{H}\right\rangle d \operatorname{vol}_{g}
\end{aligned}
$$

where $\rho_{t}(\vec{x}):=\max \left\{\rho(\vec{x})=\left|\vec{x}-\vec{x}^{0}\right|, t\right\}$. We write

$$
\begin{gathered}
\int_{M \cap B_{T}\left(\vec{x}^{0}\right) \backslash B_{t}\left(\vec{x}^{0}\right)} \frac{\left|\left(\vec{x}-\vec{x}^{0}\right)^{\perp}\right|^{2}}{\left|\vec{x}-\vec{x}^{0}\right|^{4}} d \operatorname{vol}_{g} \\
=\int_{M \cap B_{T}\left(\vec{x}^{0}\right) \backslash B_{t}\left(\vec{x}^{0}\right)}\left|\frac{\left(\vec{x}-\vec{x}^{0}\right)}{\rho^{2}}+\frac{\vec{H}}{2}\right|^{2} d \operatorname{vol}_{g} \\
-\frac{1}{4} \int_{M \cap B_{T}\left(\vec{x}^{0}\right) \backslash B_{t}\left(\vec{x}^{0}\right)}|\vec{H}|^{2} \\
-\int_{M \cap B_{T}\left(\vec{x}^{0}\right) \backslash B_{t}\left(\vec{x}^{0}\right)} \frac{\left\langle\vec{x}-\vec{x}^{0}, \vec{H}\right\rangle}{\rho^{2}} d \operatorname{vol}_{g} .
\end{gathered}
$$

Combining (A.22) and (A.23) gives (A.13) and Lemma A.3 is proved.

The following lemma is more or less contained in previous works on the subject but, for the convenience of the reader, we give a proof of it.

\footnotetext{
${ }^{6}$ Formula (A.21) generalizes in dimension 2 the formula (17.3) in [14, p. 84].
} 
Lemma A.4. Let $(\Sigma, c)$ be a connected closed Riemann surface. Let $a_{1}, \ldots, a_{N}$ be a finite family of points in the surface. Let $\bar{h}$ be some smooth conformal metric on $\Sigma$ and denote

$$
\Sigma_{\delta}:=\Sigma \backslash \bigcup_{i=1}^{N} B_{\delta}^{\bar{h}}\left(a_{i}\right)
$$

where $B_{\delta}^{\bar{h}}\left(a_{i}\right)$ is the geodesic ball of center $a_{i}$ and radius $\delta$ for the metric $\bar{h}$. Assume there exists a weak conformal Lipschitz immersion $\vec{\xi}_{\infty}$ of $\Sigma$ into $\mathbb{R}^{m}$ with $L^{2}$-bounded second fundamental form (i.e. $\vec{\xi}_{\infty}$ is an element of $\mathcal{E}_{\Sigma}^{c}$ ) and assume there exists a sequence of weak conformal Lipschitz immersions $\vec{\xi}_{k}$ of $\Sigma_{\delta}$ into $\mathbb{R}^{m}$ (for any $\delta>0$ provided $k$ is large enough) such that

$$
\forall \delta>0, \quad \vec{\xi}_{k} \rightarrow \vec{\xi}_{\infty} \quad \text { weakly in } W^{2,2} \cap\left(W^{1, \infty}\right)^{*}\left(\Sigma_{\delta}\right)
$$

and

$$
\forall \delta>0, \quad \limsup _{k \rightarrow+\infty}\left\|\log \left|d \vec{\xi}_{k}\right|_{h}\right\|_{L^{\infty}\left(\Sigma_{\delta}\right)}
$$

where the different norms $W^{2,2}, W^{1, \infty}$ and $L^{\infty}$ are taken with respect to the $\bar{h}$ metric. We assume $\vec{\xi}_{\infty}\left(a_{i}\right) \neq \vec{\xi}_{\infty}\left(a_{j}\right)$ for $i \neq j$. Let $x_{k}$ be a sequence of points such that $x_{k} \notin \vec{\xi}_{k}\left(\Sigma_{\delta}\right)$ for any $\delta>0$ for $k$ large enough and converging to $\vec{\xi}_{\infty}\left(a_{1}\right)$. Denote by $I_{x_{k}}$ the inversion with respect to the point $x_{k}$, i.e.

$$
I_{x_{k}}(x):=x_{k}+\frac{x-x_{k}}{\left|x-x_{k}\right|^{2}} .
$$

Then the following inequality holds:

$$
\lim _{\delta \rightarrow 0} \liminf _{k \rightarrow+\infty} W\left(I_{x_{k}} \circ \vec{\xi}_{k}\left(\Sigma_{\delta}\right)\right) \geq W\left(\vec{\xi}_{\infty}\right)-4 \pi
$$

Assume now that the sequence of points either diverges to $\infty$ or converges to a point $x_{\infty} \notin \vec{\xi}_{\infty}(\Sigma)$. Then

$$
\lim _{\delta \rightarrow 0} \liminf _{k \rightarrow+\infty} W\left(I_{x_{k}} \circ \vec{\xi}_{k}\left(\Sigma_{\delta}\right)\right) \geq W\left(\vec{\xi}_{\infty}\right)
$$

Proof. Denote by $\ln g_{k}:=\vec{\xi}_{k}^{*} g_{\mathbb{R}^{m}}$ the metric on $\Sigma_{\delta}$ induced by the immersion $\vec{\xi}_{k}$. Since $I_{x_{k}}$ is a conformal diffeomorphism from $\mathbb{R}^{m} \cup\{\infty\}$ into itself, the induced metric

$$
\tilde{g}_{k}:=\left(I_{x_{k}} \circ \vec{\xi}_{k}\right)^{*} g_{\mathbb{R}^{m}}=\vec{\xi}_{k}^{*}\left(I_{x_{k}}^{*} g_{\mathbb{R}^{m}}\right)
$$

is conformally equivalent to $g_{k}$. One has $I_{x_{k}}^{*} g_{\mathbb{R}^{m}}=\frac{1}{\left|x-x_{k}\right|^{4}} g_{\mathbb{R}^{m}}$, thus we obtain

$$
\tilde{g}_{k}=\frac{1}{\left|\vec{\xi}_{k}-x_{k}\right|^{4}} g_{k} \text {. }
$$


We denote by $K_{g_{k}}$ and $K_{\tilde{g}_{k}}$ the Gauss curvatures with respect to $g_{k}$ resp. $\tilde{g}_{k}$. A classical computation in the differential geometry of surfaces gives

$$
K_{g_{k}}-\frac{1}{\left|\vec{\xi}_{k}-x_{k}\right|^{4}} K_{\tilde{g}_{k}}=-\Delta_{g_{k}} \log \left|\vec{\xi}_{k}-x_{k}\right|^{2} .
$$

The integration of this identity over $\Sigma_{\delta}$ with respect to the $g_{k}$ volume form gives

$$
\begin{aligned}
\int_{\Sigma_{\delta}} K_{g_{k}} d \operatorname{vol}_{g_{k}}-\int_{\Sigma_{\delta}} K_{\tilde{g}_{k}} d \operatorname{vol}_{\tilde{g}_{k}} & =-\int_{\Sigma_{\delta}} \Delta_{g_{k}} \log \left|\vec{\xi}_{k}-x_{k}\right|^{2} d \operatorname{vol}_{g_{k}} \\
& =\int_{\partial \Sigma_{\delta}} d \log \left|\vec{\xi}_{k}-x_{k}\right|^{2} \cdot v_{k} d l_{g_{k}}
\end{aligned}
$$

where $v_{k}$ is the unit outward normal in $\Sigma$ to $\partial \Sigma_{\delta}$ with respect to the $g_{k}$ metric and $d l_{g_{k}}$ is the length form with respect to $g_{k}$ along $\partial \Sigma_{\delta}$. Let $A_{g_{k}}^{0}$, resp. $A_{\tilde{g}_{k}}^{0}$, denote the trace free second fundamental form of $\vec{\xi}_{k}$, resp. $I_{x_{k}} \circ \vec{\xi}_{k}$. We have

$$
\left|A_{g_{k}}^{0}\right|^{2}=4\left(\left|\vec{H}_{\vec{\xi}_{k}}\right|^{2}-K_{g_{k}}\right) \quad \text { and } \quad\left|A_{\tilde{g}_{k}}^{0}\right|^{2}=4\left(\left|\vec{H}_{I_{x_{k}} \circ \vec{\xi}_{k}}\right|^{2}-K_{\tilde{g}_{k}}\right) .
$$

A classical result in conformal geometry (see [11, Theorem VI.1]) gives

$$
\left|A_{g_{k}}^{0}\right|^{2} d \operatorname{vol}_{g_{k}}=\left|A_{\tilde{g}_{k}}^{0}\right|^{2} d \operatorname{vol}_{\tilde{g}_{k}} .
$$

Hence we have

$$
\left|\vec{H}_{\vec{\xi}_{k}}\right|^{2} d \operatorname{vol}_{g_{k}}-K_{\vec{\xi}_{k}} d \operatorname{vol}_{g_{k}}=\left|\vec{H}_{I_{x_{k}} \circ \vec{\xi}_{k}}\right|^{2} d \operatorname{vol}_{\tilde{g}_{k}}-K_{I_{x_{k}} \circ \vec{\xi}_{k}} d \operatorname{vol}_{\tilde{g}_{k}}
$$

Integrating this identity over $\Sigma_{\delta}$ and combining this with (A.26) gives

$$
\int_{\Sigma_{\delta}}\left|\vec{H}_{\vec{\xi}_{k}}\right|^{2} d \operatorname{vol}_{g_{k}}-\int_{\Sigma_{\delta}}\left|\vec{H}_{I_{x_{k}} \circ \vec{\xi}_{k}}\right|^{2} d \operatorname{vol}_{\tilde{g}_{k}}=\int_{\partial \Sigma_{\delta}} d \log \left|\vec{\xi}_{k}-x_{k}\right|^{2} \cdot v_{k} d l_{g_{k}} .
$$

Then [13, Lemma A.5 of] gives an asymptotic expansion of $\vec{\xi}_{\infty}$ in a neighborhood of $a_{1}$ from which we deduce that

$$
\operatorname{dist}\left(\vec{\xi}_{\infty}\left(\partial B_{\delta}\left(a_{1}\right)\right), \vec{\xi}_{\infty}\left(a_{1}\right)\right) \geq C_{\delta}>0
$$

for some positive constant $C_{\delta}$. Since $x_{k}$ converges to $\vec{\xi}_{\infty}$ and since $\vec{\xi}_{k}$ converges in $C^{0}$-norm to $\vec{\xi}_{\infty}$ on $\Sigma_{\delta}$, we have that for any $\delta>0$ there exists $c_{\delta}>0$ such that, for $k$ large enough

$$
\operatorname{dist}\left(\vec{\xi}_{k}\left(\partial B_{\delta}\left(a_{1}\right)\right), x_{k}\right) \geq c_{\delta}>0 .
$$

Since $\vec{\xi}_{k}$ weakly converges in $W^{2,2}$-norm towards $\vec{\xi}_{\infty}$ on $\Sigma_{\delta}$, $d \vec{\xi}_{k}$ strongly converges towards $d \vec{\xi}_{\infty}$ in $L^{p}\left(\Sigma_{\delta}\right)$ for any $p<+\infty$. In $\Sigma_{\delta} \backslash \Sigma_{2 \delta}$ we denote by $\bar{v}$ 
the vector-field equal to the outward unit normal to $\partial \Sigma_{s}$ for $s \in(\delta, 2 \delta)$ for the metric $\bar{h}$. Since $g_{k}$ is conformally equivalent to $\bar{h}$, we have that $v_{k} d l_{g_{k}}=\bar{v} d l_{\bar{h}}$ and we have for $s \in(\delta, 2 \delta)$

$$
\int_{\partial \Sigma_{s}} d \log \left|\vec{\xi}_{k}-x_{k}\right|^{2} \cdot v_{k} d l_{g_{k}}=\int_{\partial \Sigma_{s}} d \log \left|\vec{\xi}_{k}-x_{k}\right|^{2} \cdot \bar{v} d l_{\bar{h}}
$$

Using now the strong convergence of $d \vec{\xi}_{k}$ towards $d \vec{\xi}_{\infty}$ in $L^{p}\left(\Sigma_{\delta}\right)$ for $p=2$ together with (A.28), with the $C^{0}$-convergence of $\vec{\xi}_{k}$ towards $\vec{\xi}_{\infty}$ and with the help of Fubini's theorem, we deduce that for almost every $s \in(\delta, 2 \delta)$ there exists a subsequence still denoted by $\vec{\xi}_{k}$ such that

$$
\lim _{k \rightarrow+\infty} \int_{\partial \Sigma_{s}} d \log \left|\vec{\xi}_{k}-x_{k}\right|^{2} \cdot \bar{v} d l_{\bar{h}}-\int_{\partial \Sigma_{s}} d \log \left|\vec{\xi}_{\infty}-\vec{\xi}_{\infty}\left(a_{1}\right)\right|^{2} \cdot \bar{v} d l_{\bar{h}}=0
$$

As before, since $g_{\infty}:=\vec{\xi}_{\infty}^{*} g_{\mathbb{R}^{m}}$ is conformally equivalent to $\bar{h}$, we have

$$
\int_{\partial \Sigma_{s}} d \log \left|\vec{\xi}_{\infty}-\vec{\xi}_{\infty}\left(a_{1}\right)\right|^{2} \cdot \bar{v} d l_{\bar{h}}=\int_{\partial \Sigma_{s_{k}}} d \log \left|\vec{\xi}_{\infty}-\vec{\xi}_{\infty}\left(a_{1}\right)\right|^{2} \cdot v_{\infty} d l_{g_{\infty}}
$$

For all $i \neq 1,\left|\vec{\xi}_{\infty}-\vec{\xi}_{\infty}\left(a_{1}\right)\right| \geq c>0$ on $\partial B_{S}\left(a_{i}\right)$ and, since $\vec{\xi}_{\infty}$ is Lipschitz, we easily get that

$$
\forall i \neq 1, \quad \lim _{s \rightarrow 0} \int_{\partial B_{s}\left(a_{i}\right)} d \log \left|\vec{\xi}_{\infty}-\vec{\xi}_{\infty}\left(a_{1}\right)\right|^{2} \cdot v_{\infty} d l_{g_{\infty}}=0 .
$$

At this stage, if $\vec{\xi}_{\infty}$ would be a smooth immersion, we could easily pass to the limit $s \rightarrow 0$ for $i=1$ as well and prove that this boundary integral generates a residue equal to $4 \pi$. In the case of weak immersion $\vec{\xi}_{\infty} \in \mathcal{E}_{\Sigma}$ this passage to the limit does not necessarily holds and we have to pick some well chosen sequence $s_{i}$.

We take conformal coordinates $x=\left(x_{1}, x_{2}\right)$ around $a_{1}$ such that

$$
x\left(a_{1}\right)=y\left(a_{1}\right)=0 .
$$

We assume to simplify notations that $\vec{\xi}_{\infty}\left(a_{1}\right)=0$. Let $f$ be the inverse of these coordinates. We assume to simplify the presentation that our metric $\bar{h}$ coincides with the flat metric in these coordinates. Let $\lambda_{\infty}$ be the conformal factor associated to $f^{*} g_{\infty}$, i.e. $e^{2 \lambda_{\infty}}\left[d x_{1}^{2}+d x_{2}^{2}\right]=f^{*} g_{\infty}$. By an abuse of notation we keep denoting by $\vec{\xi}_{\infty}$ the composition of $\xi_{\infty}$ with $f$. Let

$$
\vec{e}_{1}:=e^{-\lambda \infty} \partial_{x_{1}} \vec{\xi}_{\infty} \quad \text { and } \quad \vec{e}_{2}:=e^{-\lambda_{\infty}} \partial_{x_{2}} \vec{\xi}_{\infty} .
$$

For any radius $\rho>0$ we denote

$$
{\overrightarrow{\xi_{\infty}}}^{\rho}:=\left|\partial B_{\rho}(0)\right|^{-1} \int_{\partial B_{\rho}(0)} \vec{\xi}_{\infty} .
$$


We have

$$
\begin{aligned}
{\overrightarrow{\vec{\xi}_{\infty}}}^{\rho} & =\frac{1}{2 \pi \rho} \int_{\partial B_{\rho}(0)} \int_{0}^{\rho} \frac{\partial \vec{\xi}_{\infty}}{\partial r} d r \\
& =\frac{1}{2 \pi} \int_{0}^{2 \pi} \int_{0}^{\rho} \frac{e^{\lambda \infty}}{|x|}\left[\cos \theta \vec{e}_{1}+\sin \theta \vec{e}_{2}\right] r d r d \theta .
\end{aligned}
$$

For such a $\vec{\xi}_{\infty} \in \mathcal{E}_{\Sigma}, \lambda$ is continuous (see $[4,11]$ for instance). Hence we have

$$
\int_{B_{\rho}(0)} \frac{\mid e^{\lambda \infty}-e^{\lambda_{\infty}(0) \mid}}{|x|}=o(1) \rho .
$$

For $i=1,2$ we denote

$$
\vec{e}_{i}^{\rho}:=\left|\partial B_{\rho}(0)\right|^{-1} \int_{\partial B_{\rho}(0)} \vec{e}_{i} .
$$

Since $\vec{e}_{i}$ is $W^{1,2}$, we have by using the Hölder and Sobolev-Poincaré inequalities

$$
\begin{aligned}
\int_{B_{\rho}(0)} \frac{\left|\vec{e}_{i}-\vec{e}_{i}^{\rho}\right|}{|x|} & \leq C \rho\left[\frac{1}{\left|B_{\rho}(0)\right|} \int_{B_{\rho}(0)}\left|\vec{e}_{i}-\vec{e}_{i}^{\rho}\right|^{3}\right]^{1 / 3} \\
& \leq C \rho\left[\int_{B_{\rho}(0)}\left|\nabla \vec{e}_{i}\right|^{2}\right]^{1 / 2}=\rho o(1) .
\end{aligned}
$$

Since

$$
\int_{0}^{2 \pi} \cos \theta \vec{e}_{1}^{\rho}+\sin \theta \vec{e}_{2}^{\rho}=0
$$

combining (A.32), (A.33) and (A.34) gives

$$
\left|\overline{\vec{\xi}}_{\infty}^{\rho}\right| \leq \rho o(1) \text {. }
$$

Let

$$
\vec{X}_{i}^{\rho}:=\left|B_{\rho}(0)\right|^{-1} \int_{B_{\rho}(0)} \partial_{x_{i}} \vec{\xi}_{\infty}=\left|B_{\rho}(0)\right|^{-1} \int_{B_{\rho}(0)} e^{\lambda_{\infty} \vec{e}_{i}}
$$

Using Poincaré inequality, we have

$$
\frac{1}{\left|B_{\rho}(0)\right|} \int_{B_{\rho}(0)}\left|\partial_{x_{i}} \vec{\xi}_{\infty}-\vec{X}_{i}^{\rho}\right|^{2} \leq C \int_{B_{\rho}(0)}\left|\nabla^{2} \vec{\xi}_{\infty}\right|^{2}=o(1)
$$

Thus there exists $x \in B_{\rho}(0)$ such that

$$
\left|\partial_{x_{i}} \vec{\xi}_{\infty}-\vec{X}_{i}^{\rho}\right|^{2}=o(1) .
$$

Since $\partial_{x_{i}} \vec{\xi}_{\infty}=e^{\lambda \infty} \vec{e}_{i}=e^{\lambda \infty(0)} \vec{e}_{i}+o(1)$, one has

$$
\left|\vec{X}_{i}^{\rho}\right|=e^{\lambda \infty}+o(1) \text {. }
$$


We have moreover

$$
\begin{aligned}
\left|\vec{X}_{1}^{\rho} \cdot \vec{X}_{2}^{\rho}\right| & =\mid \frac{1}{\left|B_{\rho}(0)\right|^{2}} \int_{B_{\rho}(0)} \int_{B_{\rho}(0)} \partial_{x_{1}} \vec{\xi}_{\infty}(x) \cdot \partial_{x_{2}} \vec{\xi}_{\infty}(y) \\
& \quad-\partial_{x_{1}} \vec{\xi}_{\infty}(y) \cdot \partial_{x_{2}} \vec{\xi}_{\infty}(y) d x_{1} d x_{2} d y_{1} d y_{2} \mid \\
\leq & \left.\frac{\left\|\nabla \vec{\xi}_{\infty}\right\|_{\infty}}{\left|B_{\rho}(0)\right|} \int_{B_{\rho}(0)}\left|\nabla \vec{\xi}_{\infty}-\right| B_{\rho}(0)\right|^{-1} \int_{B_{\rho}(0)} \nabla \vec{\xi}_{\infty} \mid \\
\leq & C \int_{B_{\rho}(0)}\left|\nabla^{2} \vec{\xi}_{\infty}\right|=o(1) .
\end{aligned}
$$

On $B_{\rho}(0)$ we define $\vec{u}_{\rho}:=\vec{\xi}_{\infty}-x_{1} \vec{X}_{1}^{\rho}-x_{2} \vec{X}_{1}^{\rho}$. Using again Poincaré's inequality, we have

$$
\begin{aligned}
\int_{B_{\rho}(0)}\left|\nabla u_{\rho}\right|^{2} & =\left.\left.\int_{B_{\rho}(0)}\left|\nabla \vec{\xi}_{\infty}-\right| B_{\rho}(0)\right|^{-1} \int_{B_{\rho}(0)} \nabla \vec{\xi}_{\infty}\right|^{2} \\
& \leq C \rho^{2} \int_{B_{\rho}(0)}\left|\nabla^{2} \vec{\xi}_{\infty}\right|^{2}=o(1) \rho^{2}
\end{aligned}
$$

Using the Hölder inequality, Fubini's theorem and the mean value formula, we deduce from (A.39) that there exists $s \in(\rho / 2, \rho)$ such that

$$
\left\{\begin{array}{l}
\left\|u_{\rho}-\bar{u}_{\rho}^{s}\right\|_{L^{\infty}\left(\partial B_{S}(0)\right)} \leq \int_{\partial B_{S}(0)}\left|\nabla u_{\rho}\right| \leq o(1) s, \\
\int_{\partial B_{S}(0)}\left|\partial_{x_{i}} \vec{\xi}_{\infty}-\vec{X}_{i}^{\rho}\right|^{2}=o(1) s \text { for } i=1,2 .
\end{array}\right.
$$

Then (A.35) gives that

$$
\left|\bar{u}_{\rho}^{s}\right|=o(1) s .
$$

Hence from (A.37), (A.38) and (A.40), proceeding to a classical Schmidt orthonormalization of $e^{-\lambda_{\infty}(0)}\left(\vec{X}_{1}^{\rho}, \vec{X}_{1}^{\rho}\right)$, we deduce that there exists a radius $s \in(\rho / 2, \rho)$ and a pair of unit vectors $\left(\vec{f}_{1}^{\rho}, \vec{f}_{2}^{\rho}\right)$ orthogonal with another such that

$$
e^{-\lambda_{\infty}(0)}\left(\vec{X}_{1}^{\rho}, \vec{X}_{1}^{\rho}\right)=\left(\vec{f}_{1}^{\rho}, \vec{f}_{2}^{\rho}\right)+o(1)
$$

and

$$
\left\|\vec{\xi}_{\infty}-e^{\lambda \infty(0)}\left[x_{1} \vec{f}_{1}^{\rho}-x_{2} \vec{f}_{2}^{\rho}\right]\right\|_{L^{\infty}\left(\partial B_{S}(0)\right)}=o(1) s .
$$

This implies in particular that

$$
\left\|\left|\vec{\xi}_{\infty}\right|-e^{\lambda \infty(0)}|x|\right\|_{L^{\infty}\left(\partial B_{S}(0)\right)}=o(1) s .
$$


Combining (A.43), (A.41) and the second line of (A.40) gives

$$
\begin{aligned}
\int_{\partial B_{s}\left(a_{1}\right)} d \log \left|\vec{\xi}_{\infty}-\vec{\xi}_{\infty}\left(a_{1}\right)\right|^{2} \cdot v_{\infty} d l_{g_{\infty}} & =2 s \int_{0}^{\theta} \frac{\partial \vec{\xi}_{\infty}}{\partial r} \cdot \frac{\vec{\xi}_{\infty}}{\left|\vec{\xi}_{\infty}\right|^{2}} d \theta \\
& =4 \pi+o(1) .
\end{aligned}
$$

Thus combining (A.27), (A.31) and (A.44), for this special choice of $s \in(\delta / 2, \delta)$ we have found a subsequence still denoted by $\vec{\xi}_{k}$ such that

$$
\begin{aligned}
\liminf _{k \rightarrow+\infty} & \int_{\Sigma_{s}}\left|\vec{H}_{I_{x_{k}} \circ \vec{\xi}_{k}}\right|^{2} d \operatorname{vol}_{\tilde{g}_{k}} \\
& \geq \liminf _{k \rightarrow+\infty} \int_{\Sigma_{s}}\left|\vec{H}_{\vec{\xi}_{k}}\right|^{2} d \operatorname{vol}_{g_{k}}-4 \pi+o_{\delta}(1) .
\end{aligned}
$$

Since

$$
\liminf _{k \rightarrow+\infty} \int_{\Sigma_{s}}\left|\vec{H}_{\vec{\xi}_{k}}\right|^{2} d \operatorname{vol}_{g_{k}} \geq \int_{\Sigma_{s}}\left|\vec{H}_{\vec{\xi}_{\infty}}\right|^{2} d \operatorname{vol}_{g_{\infty}},
$$

we have proved that for all $\varepsilon>0$ there exists $s>0$ and a subsequence still denoted by $\vec{\xi}_{k}$ such that

$$
\liminf _{k \rightarrow+\infty} W\left(I_{x_{k}} \circ \vec{\xi}_{k}\left(\Sigma_{s}\right)\right) \geq W\left(\vec{\xi}_{\infty}\left(\Sigma_{s}\right)\right)-4 \pi-\varepsilon .
$$

Using a diagonal argument, we can choose a unique subsequence $\vec{\xi}_{k}$ such that formula (A.44) holds for $\delta=2^{-j}$ for each $j \in \mathbb{N}$ and with a well-chosen element $s_{j} \in\left(2^{-j-1}, 2^{-j}\right)$. Since $\liminf _{k \rightarrow+\infty} W\left(I_{x_{k}} \circ \vec{\xi}_{k}\left(\Sigma_{s}\right)\right)$ is a decreasing function of $\delta$, we have proved that

$$
\lim _{\delta \rightarrow 0} \liminf _{k \rightarrow+\infty} W\left(I_{x_{k}} \circ \vec{\xi}_{k}\left(\Sigma_{\delta}\right)\right) \geq W\left(\vec{\xi}_{\infty}(\Sigma)\right)-4 \pi-\varepsilon .
$$

Hence we have proved that from any sequence satisfying the assumption of the lemma one can extract a subsequence such that (A.48) holds; we have then proved (A.24). The last case when no subsequence of $x_{k}$ converge to a point of $\vec{\xi}_{\infty}(\Sigma)$ is covered by the previous analysis since the problem of the limiting point $\vec{\xi}_{\infty}\left(a_{1}\right)$ as being an accumulation point of $x_{k}$ does not show up and one has (A.25) in that case. Lemma A. 4 is then proved.

\section{Bibliography}

[1] M. Bauer and E. Kuwert, Existence of minimizing Willmore surfaces of prescribed genus, Int. Math. Res. Not. IMRN 2003 (2003), no. 10, 553-576.

[2] Y. Bernard and T. Rivière, Local Palais-Smale sequences for the Willmore functional, preprint (2009), http://arxiv .org/abs/0904.0360. 
[3] Y. Bernard and T. Rivière, Energy quantization for Willmore surfaces and applications, preprint (2011), http: //arxiv.org/abs/1106.3780.

[4] F. Hélein, Harmonic Maps, Conservation Laws, and Moving Frames, Cambridge Tracts in Mathematics 150, Cambridge University Press, Cambridge, 2002.

[5] C. Hummel, Gromov's Compactness Theorem for Pseudo-Holomorphic Curves, Progress in Mathematics 151, Birkhäuser-Verlag, Basel, 1997.

[6] E. Kuwert and Y. Li, $W^{2,2}$-conformal immersions of a closed Riemann surface into $\mathbb{R}^{n}$, preprint (2010), http: //arxiv .org/abs/1007.3967.

[7] E. Kuwert, Y. Li and R. Schätzle, The large genus limit of the infimum of the Willmore energy, Amer. J. Math. 132 (2010), no. 1, 37-51.

[8] E. Kuwert and R. Schätzle, Removability of point singularities of Willmore surfaces, Ann. of Math. (2) 160 (2004), no. 1, 315-357.

[9] E. Kuwert and R. Schätzle, Closed surfaces with bounds on their Willmore energy, preprint, Centro di Ricerca Matematica Ennio De Giorgi, Pisa, 2008.

[10] S. Müller and V. Šverák, On surfaces of finite total curvature, J. Differential Geom. 42 (1995), no. 2, 229-258.

[11] T. Rivière, Conformally invariant 2-dimensional variational problems, Cours joint de l'Institut Henri Poincaré, Paris XII Creteil, Novembre 2010.

[12] T. Rivière, Analysis aspects of Willmore surfaces, Invent. Math. 174 (2008), no. 1, $1-45$.

[13] T. Rivière, Variational principles for immersed surfaces with $L^{2}$-bounded second fundamental form, preprint (2010), http://arxiv.org/abs/1007.2997.

[14] L. Simon, Lectures on Geometric Measure Theory, Australian National University Centre for Mathematical Analysis, Canberra, 1983.

[15] L. Simon, Existence of surfaces minimizing the Willmore functional, Comm. Anal. Geom. 1 (1993), no. 2, 281-326.

[16] M. Zhu, Harmonic maps and Dirac-harmonic maps from Degenerating Type, $\mathrm{PhD}$ thesis, Leipzig University, 2008.

Received June 17, 2011; revised November 2, 2011; accepted November 8, 2011.

\section{Author information}

Tristan Rivière, Department of Mathematics, ETH Zentrum, CH-8093 Zürich, Switzerland. 